\title{
Surface properties of simple metals via inhomogeneous linear electronic response: I. Theory
}

\author{
J F Dobson $\uparrow+$ and J H Rose $\$$ \\ + Physics Department, University of Michigan, Ann Arbor, Michigan, 48109, USA \\ $\ddagger$ School of Science, Griffith University, Nathan, Queensland, 4111, Australia|| \\ $\S$ Ames Laboratory, Iowa State University, Ames, Iowa, 50011, USA
}

Received 27 April 1982, in final form 28 July 1982

\begin{abstract}
This paper is the first of a series addressing the macroscopic surface properties of the simple metals (the alkalis, $\mathrm{Mg}, \mathrm{Zn}, \mathrm{Al}, \mathrm{Pb}$ ) using pseudopotential perturbation theory to include the effects of discrete ions beyond the first-order Lang-Kohn jellium calculation. Here we give explicit formulae for the self-consistently screened inhomogeneous electron susceptibility $\chi\left(q, z, z^{\prime}\right)$ calculated around the jellium surface results of Lang and Kohn. In terms of $\chi$ we then give explicit formulae, up to second order in the ionic pseudopotentials, for: (i) the energy of a bounded metal; (ii) the effective ion and ion-ion potentials near a surface; (iii) the unreconstructed surface formation energy $\sigma$ at $T=0 \mathrm{~K}$; (iv) the surface dynamical matrix; (v) the relaxation of ionic layer positions due to the surface; (vi) approximate $T \neq 0 \mathrm{~K}$ contributions to the surface free energy in the solid phase.

As tested on face-dependent surface energies, our second-order method is a decided improvement on first-order theories, and on one-dimensional variational theories. It has a further advantage over variational approaches in that expressions for (iv), (v) and (vi) can be given in analytic form.
\end{abstract}

\section{Introduction}

Many properties of simple-metal surfaces are strongly influenced by electronic screening of the ion cores. Such properties include the surface free energy (especially its face and temperature dependence), surface relaxation and reconstruction, surface vibrational frequencies, the energy (and existence) of surface electronic states, and chemisorption bonding energies.

In this series of papers we calculate some of these surface quantities, assuming that the conduction-electron-ion interactions are adequately represented by a weak pseudopotential which drives a screened linear response of the electron density. The linear response function is then used to compute the energy of the system up to second order in the pseudopotential. (Strictly, up to second order in the difference between the total pseudopotential and the potential due to a locally uniform jellium background.)

This pseudopotential perturbation approach has been widely used in the context of bulk simple metals, for which the linear response function (i.e. the screened bulk electron susceptibility $\chi^{\text {bulk }}\left(\boldsymbol{r}-\boldsymbol{r}^{\prime}\right)$ ) is well known from the theory of the uniform electron

$\|$ Permanent address. 
gas: see for example Ashcroft and Langreth (1967), Hammerberg and Ashcroft (1974) and Finnis (1974). In the case of a metal surface of the zeroth-order problem, about which the linear response is calculated, is spatially non-uniform, necessitating a translationally non-invariant response function $\chi^{\text {surfacc }}\left(r, r^{\prime}\right)$. (Finnis (1975) has shown that a bulk $\chi$ is inadequate.) Our theory takes the broken symmetry explicitly into account, using Kohn-Sham density functional theory to provide the self-consistently screened electronic susceptibility $\chi^{\text {surface }}$. The Kohn-Sham approach to linear response in inhomogeneous systems was first used by Stott and Zaremba (1980) in the context of atomic polarisabilities, with Kohn-Sham atomic wavefunctions describing the zeroth-order system.

For the surface susceptibility problem which we solve, the zeroth-order wavefunctions are the Lang-Kohn (1970) self-consistent wavefunctions for a one-dimensional 'jellium' surface (i.e. one without discrete ions). Our expression for $\chi^{\text {surface }}$ is derived in the Appendix, and appears to be computationally tractable, while being more realistic than previous calculations.

An important advantage of our pseudopotential perturbation approach over direct electronic variational schemes (e.g. those of Monnier and Perdew (1978) and Bohnen and Ying $(1979,1980)$ ) is that closed expressions in terms of $\chi$ can be obtained for quantities involving the displacements of the ions from the perfect surface configuration.

In the first paper (I) we develop a detailed theory of the energetics of simple-metal surfaces, including zero-temperature surface energies, relaxation of ionic positions, surface dynamical matrix, and thermal vibrational free energy. We do not at this stage discuss the surface electron states or chemisorption energies mentioned in the first paragraph, since these presumably require a non-perturbative treatment of the discrete ion effects.

In paper II of this series we will report in detail our numerical results for the facedependent $T=0 \mathrm{~K}$ surface energies of $\mathrm{Li}, \mathrm{Na}, \mathrm{K}, \mathrm{Rb}, \mathrm{Cs}, \mathrm{Mg}, \mathrm{Zn}, \mathrm{Pb}$ and Al. A brief report has already been published (Rose and Dobson 1981). Despite our use, so far, of a simple (but non-trivial) ansat $z$ for $\chi^{\text {surface }}$, our numbers appear to be the best obtained to date for the dependence of surface energy on exposed crystallographic face, for the range of metals considered.

In further papers of the series we intend to give numerical results for the full Kohn-Sham surface susceptibility $\chi^{\text {surface }}$, and for the other surface quantities whose formulae are derived in the present paper in terms of $\chi^{\text {surface }}$.

The present paper, I, is organised as follows:

Section 2 introduces the linear response theory of strongly non-uniform electron systems up to second order in $\Delta V_{\mathrm{ps}}$, the difference between the total pseudopotential and the potential $V_{+}^{\text {ell }}(\boldsymbol{r})$ due to an appropriate jellium background. We obtain an expression for the total energy and show that the changes in energy up to second order due to displacements of the ions can be expressed in terms of effective one-ion potentials $w(X)$ and self-consistently screened ion-ion pair potentials $\varphi^{\text {scr }}\left(\boldsymbol{X}, \boldsymbol{X}^{\prime}\right)$. The latter are not merely functions of the displacement $\boldsymbol{X}-\boldsymbol{X}^{\prime}$, for ions near the surface. We give detailed microscopic expressions for $w$ and $\varphi^{\mathrm{ser}}$ which provide an ab initio basis for force-constant models of metal surfaces.

Section 3 sets up the $T=0 \mathrm{~K}$ surface energy problem in a consistent fashion, starting from the electronically relaxed second-order bulk metal and computing energy changes step-by-step until the electronically relaxed second-order surface system is attained. The first-order Lang-Kohn (1970) surface energy $\sigma^{\mathrm{LK}}$ appears as the energy involved in one of our steps. The problem of numerically subtracting large surface and bulk terms 
to obtain a small surface energy is avoided by a careful analytical combination of our terms.

Section 4 considers relaxation of ionic layer positions in a direction perpendicular to the surface. Any number of layers can be relaxed analytically within our lattice statics formulation. We do not consider intralayer reconstruction in this section.

In $\S 5$ we obtain expression for the layer dynamical matrix $D_{\alpha \beta}\left(\boldsymbol{q}_{\|}, l, l^{\prime}\right)$ appropriate to phonon calculations for metal surfaces. This can be used to study surface reconstruction, and in principle also to calculate surface thermodynamic properties.

Section 6 discusses Einstein and self-consistent Einstein approximations which promise to simplify the calculation of integrated phonon properties such as temperature-dependent surface free energies and thermal expansion. A first-principles study of temperature-dependent reconstruction appears possible by combining the results of this section with those of $\S 5$.

The Appendix contains a discussion of self-consistently screened surface response functions $\chi\left(q_{\|}, z, z^{\prime}\right)$.

\section{Energy of bounded metal: linear pseudopotential response}

It is convenient to refer the energy of the bounded metal to that of a comparable 'jellium' system. In jellium, the positive ions are replaced by a half-space of uniform charge, with charge density

$$
|e| n_{+}^{\text {jell }}(r)=|e| n_{0} \theta(-z),
$$

and the total unscreened ionic pseudopotential $V_{\mathrm{ps}}(r)$ is replaced by the potential $V_{+}^{j e l l}(r)$ due to the density (2.1). When the discrete ions are put back in, the electrons feel a change in unscreened, external potential equal to

$$
\Delta V_{\mathrm{ps}}(\boldsymbol{r})=V_{\mathrm{ps}}(\boldsymbol{r})-V_{+}^{\text {jell }}(\boldsymbol{r})
$$

We define the (self-consistently screened) linear electronic susceptibility, $\chi\left(r, r^{\prime}\right)$, of the bounded jellium system in terms of the linear change in electronic number density,

$$
\Delta n_{-}(\boldsymbol{r})=n_{-}(\boldsymbol{r})-n_{-}^{\text {jell }}(\boldsymbol{r}),
$$

which is induced by an arbitrary perturbation $\Delta V_{\mathrm{ps}}(\boldsymbol{r})$ : thus

$$
\Delta n_{-}(r)=\int \chi\left(r, r^{\prime}\right) \Delta V_{\mathrm{ps}}\left(\boldsymbol{r}^{\prime}\right) \mathrm{d}^{3} \boldsymbol{r}^{\prime}+\mathrm{O}\left(\left(\Delta V_{\mathrm{ps}}\right)^{2}\right) .
$$

We stress that $\chi$ includes self-consistent screening. The symbol $\chi$ will refer to the bounded metal except as noted.

We now perform a Feynman integration with respect to the electronic part of the Hamiltonian. (That is, we replace $\Delta V_{p s}$ by $\lambda \Delta V_{p s}$ and use the Feynman-Hellman theorem to compute the change in total energy as $\lambda$ varies from 0 to 1.) This gives the following expression for the total energy of a set of discrete stationary ions at positions $\{\boldsymbol{X}(l)\}$, plus interacting conduction electrons:

$$
\begin{aligned}
E=E^{\text {jell }}-E_{+}^{\text {jell }} & +\frac{1}{2} \sum_{l \neq l^{\prime}} v^{\text {direct }}\left(\boldsymbol{X}(\boldsymbol{l})-\boldsymbol{X}\left(\boldsymbol{l}^{\prime}\right)\right)+\int n_{-}^{\mathrm{jell}}(\boldsymbol{r}) \Delta V_{\mathrm{ps}}(\boldsymbol{r}) \mathrm{d}^{3} \boldsymbol{r} \\
& +\frac{1}{2} \int \Delta V_{\mathrm{ps}}(\boldsymbol{r}) \chi\left(\boldsymbol{r}, \boldsymbol{r}^{\prime}\right) \Delta V_{\mathrm{ps}}\left(\boldsymbol{r}^{\prime}\right) \mathrm{d}^{3} \boldsymbol{r} \mathrm{d}^{3} \boldsymbol{r}^{\prime}+\mathrm{O}\left(\left(\Delta V_{\mathrm{ps}}\right)^{3}\right) .
\end{aligned}
$$


Here $v^{\text {direct }}$ is the bare ion-ion potential energy, and $E_{++}^{\text {jell }}$ is the Coulomb self-energy of the positive jellium background. Note that all effects of $\Delta V_{\mathrm{ps}}$ on the electron kinetic as well as potential energy are included via the factor $\frac{1}{2}\left(=\int_{0}^{1} \lambda \mathrm{d} \lambda\right)$ in the last term of (2.5). Equation (2.5) is a real-space equivalent of the well known $k$-space pseudopotential (or 'structural') expansion of bulk simple metal energies (see Hammerberg and Ashcroft (1974) and references therein). An equation of the form (2.5) has been discussed by Inglesfield (1979), though his $\Delta V$ is due to displacement of ions already present in the zeroth-order system; no jellium is involved.

We will assume, as have most authors (e.g. Lang and Kohn 1970), that the unscreened pseudopotential $v^{\mathrm{ps}}$ appropriate for a single ion near the surface is the same as for an ion in the bulk. We can then write the total unscreened pseudopotential perturbation as the sum of terms due to charge-neutral entities:

$$
\Delta V_{\mathrm{ps}}(r)=\sum_{l} \Delta v^{\mathrm{ps}}(r, X(l))
$$

where

$$
\Delta v^{\mathrm{ps}}(\boldsymbol{r}, \boldsymbol{X}(l))=v^{\mathrm{ps}}(\boldsymbol{r}-\boldsymbol{X}(l))-v^{\text {block }}\left(\boldsymbol{r}-\boldsymbol{R}^{0}(l)\right) .
$$

Here $v^{\mathrm{ps}}(r)$ is the potential energy of an electron at $r$, due to a pseudo-ion at the origin. $\Delta v^{\text {block }}\left(r-\boldsymbol{R}^{0}(l)\right)$ is the potential energy of an electron at $r$, due to a block of uniform jellium background centred at a perfect lattice site $R^{0}(l)$. The blocks fit together without spaces to make the jellium background. For crystalline surface problems the blocks can conveniently be chosen to have a surface direct lattice cell as base, and a perpendicular height equal to the ionic layer spacing $d$. Then (2.5) can be written

$$
E(\{X(l)\})=E_{0}+\sum_{l} w(X(l))+\frac{1}{2} \sum_{l, l} \varphi^{s c t}\left(X(l), X\left(l^{\prime}\right)\right)
$$

where

$$
w(X(l))=\int n_{-}^{\text {jell }}(\boldsymbol{r}) \Delta v^{\mathrm{ps}}(\boldsymbol{r}, \boldsymbol{X}(l)) \mathrm{d}^{3} r
$$

is an effective one-ion potential, and

$$
\begin{aligned}
\varphi^{\mathrm{scr}}\left(\boldsymbol{X}(l), \boldsymbol{X}\left(l^{\prime}\right)\right) & =\int \chi\left(\boldsymbol{r}, \boldsymbol{r}^{\prime}\right) \Delta v^{\mathrm{ps}}(\boldsymbol{r}, \boldsymbol{X}(l)) \Delta v^{\mathrm{ps}}\left(\boldsymbol{r}^{\prime}, \boldsymbol{X}\left(l^{\prime}\right)\right) \mathrm{d}^{3} r \mathrm{~d}^{3} \boldsymbol{r}^{\prime} \\
+ & v^{\mathrm{direct}}\left(X(l), X\left(l^{\prime}\right)\right)
\end{aligned}
$$

is an effective screened ion-ion potential.

The constant $E_{0}$ is $E^{\text {jell }}-E_{++}^{\text {jell }}$; it depends on volume and shape. Note that (2.8) explicitly allows the ion $l$ to interact with itself via $\varphi^{\text {scr }}(\boldsymbol{X}(l), \boldsymbol{X}(l))$. This is a real effect: the polarisation cloud induced by an ion does interact with the ion, as expressed in the first term of (2.10). This self-interaction can be position-dependent (e.g. near a surface). Of course the direct term in (2.10) is spurious when $l=l^{\prime}$, if we take $v^{\text {direct }}$ to be the ion-ion Coulomb interaction. We can take $v^{\text {direct }}(\boldsymbol{X}(l), X(l))$ to be any finite constant, however, and this only changes the constant $E_{0}$ in (2.8); the variation of the energy with changes in $\{X(l)\}$ is unaffected, and it is convenient not to have a restriction $l \neq l^{\prime}$ in (2.8). We note also that other choices are possible for $\varphi^{\text {ser }}$ and $w$. For example, we could use $v^{\mathrm{ps}}$ instead of $\Delta v^{\mathrm{ps}}$ in (2.10) and (2.9) (see for example Finnis 1974). This would change the constant $E_{0}$ in (2.8) and also shift some terms from $\varphi^{\text {scr }}$ into $w$ : this is not a paradox as there is no sharp distinction between one- and two-body terms when we are considering self-interactions $\left(l=l^{\prime}\right)$. 
We intend to make quantitative use of equations (2.5) and (2.8)-(2.10), and thus we require accurate values of the surface susceptibility $\chi\left(r, r^{\prime}\right)$, or more conveniently its surface Fourier transform $\chi\left(q_{\|}, z, z^{\prime}\right)$. In the Appendix (equations (A31), (A37), (A38)) we give fully detailed expressions for $\chi$, as derived from Kohn-Sham local density functional theory, using Lang-Kohn (1970) jellium surface wavefunctions as input. Our expression:

(i) treats electron kinetic energy exactly;

(ii) includes electron exchange and correlation;

(iii) is self-consistently screened; and

(iv) employs a realistic smooth surface density profile.

Our method is equivalent to that of Stott and Zaremba (1980) who performed a similar calculation to obtain atomic polarisabilities. Previous detailed calculations of the surface susceptibility have either treated the kinetic energy inexactly (e.g. Ying et al 1972) or have used unrealistic step or barrier models of the surface profile (e.g. Lert and Weare 1978, Garrido et al 1979) or have omitted electron correlations or lacked selfconsistency. Inglesfield (1979) has formally introduced a quantity $F\left(r, r^{\prime}\right)$ which is our $\chi\left(r, r^{\prime}\right)$ except that his zeroth-order system (a transition metal) contains ions so that non-trivial three-dimensional zeroth-order wavefunctions are required, rendering accurate numerical computation of $\chi$ a formidable task indeed.

One of us (JFD) is currently evaluating our $\chi$ for simple metals: the numerics appear to be tractable. Pending the outcome of these computations, we would like to offer a simple ansat $z$ for $\chi$, to add to the above-mentioned simplified treatments. It is

$$
\chi^{\text {ansatz }}\left(\boldsymbol{r}, \boldsymbol{r}^{\prime}\right)=\chi^{\mathrm{bulk}}\left(\boldsymbol{r}-\boldsymbol{r}^{\prime}, \tilde{n}\left(\boldsymbol{r}, \boldsymbol{r}^{\prime}\right)\right)
$$

where

$$
\bar{n}\left(r, r^{\prime}\right)=\frac{1}{2}\left(n_{-}^{\text {jell }}(z)+n_{-}^{\text {jell }}\left(z^{\prime}\right)\right)
$$

is an 'average zeroth-order density' formed from the Lang-Kohn (1970) jellium values. $\chi^{\text {bulk }}\left(\left|\boldsymbol{r}-\boldsymbol{r}^{\prime}\right|, n\right)$ is the three-dimensional Fourier transform of the bulk (screened) Kohn-Sham susceptibility

$$
\chi^{\text {bulk }}(k, n)=\chi^{0 \mathrm{~L}}(k) /\left[1-\left(\frac{4 \pi}{k^{2}}+\frac{\partial^{2}\left(n \varepsilon_{\mathrm{xc}}\right)}{\partial n^{2}}\right) \chi^{0 \mathrm{~L}}(k)\right]
$$

where $\chi^{0 \mathrm{~L}}(k)$ is the unscreened Lindhard (1954) susceptibility of a uniform gas of density $n$.

We have used $\chi^{\text {ansatz }}$ so far in surface energy calculations (Rose and Dobson 1981), and find our results to be a qualitative improvement over previous work. Our only real justification for the average-density ansatz (2.11), however, is its success (Chakravarty et al 1981) when tested on $\mathrm{H}_{2}$ molecule calculations. It is certainly inaccurate to use the bulk density for $\bar{n}$ in equation (2.11), as shown by the work of Finnis (1975).

\section{The surface energy up to second order}

Several calculations of the surface energy of simple metals have been published to date; see, for example, Paasch and Hietschold (1977), Lang and Kohn (1970), Monnier and Perdew (1978). None of these produced satisfactory face-dependent surface energies for the whole range of simple metals (see Rose and Dobson 1981). In a recent paper, of 
which we received a preprint when the present work was substantially complete, Bohnen and Ying (1980) have used a density matrix variational method to obtain good results for the 110 and 100 faces of $\mathrm{Na}, \mathrm{K}, \mathrm{Rb}$ and $\mathrm{Cs}$. The method we describe here gives good face-dependent results across the whole range of simple metals with the possible exception of $\mathrm{Zn}$, agreeing with Bohnen and Ying's numbers for the metals they considered. We are also able to compute surface lattice statics and dynamics in a simple fashion using our method (see $\S \S 4,5$ and 6 of the present paper).

In a previous publication (Rose and Dobson 1981) we asserted that our second-order corrections for the surface and bulk systems could simply be added to and subtracted from the Lang-Kohn results, respectively. We now justify this assertion systematically. (Computational details are left to paper II of this series, however. Some preliminary numerical results, based on a simplified susceptibility $\chi$, have already been reported in Rose and Dobson (1981).)
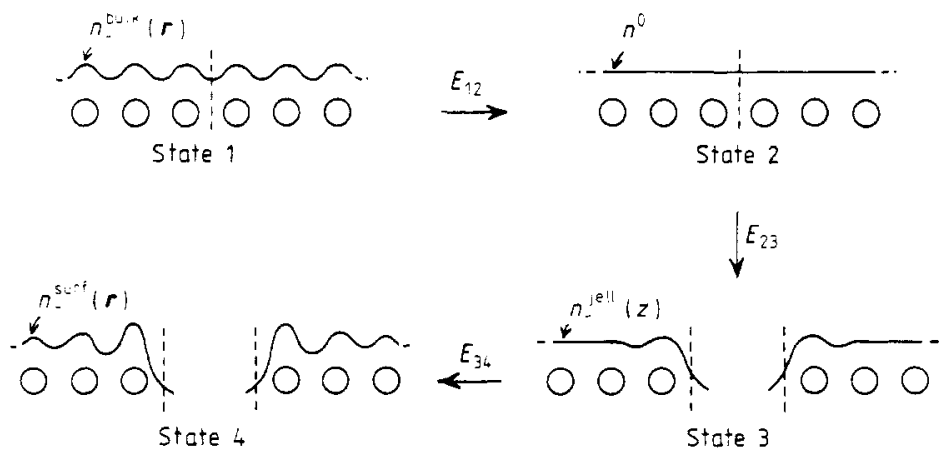

Figure 1. The steps involved in computing the formation energy of an ionically unrelaxed surface.

We start from the bulk metal and change it, in three steps, into a pair of semi-infinite metal slabs, each with area $A$. We keep track of the total energy of each step, carefully choosing our intermediate quantum states ' 2 ' and ' 3 ' so that their energy difference $E_{23}$ per unit area is exactly twice the total surface energy $\sigma^{L K}$ which Lang and Kohn (1970) computed up to first order in $\Delta V_{\mathrm{ps}}$. Figure 1 illustrates our procedure. The open circles represent static ions at perfect lattice sites. We cleave between planes separated by a spacing $d$. Broken lines represent the cleavage plane, which is distant $d / 2$ from the adjacent ion planes. The cleavage plane is drawn even in the cleaved states 3 and 4 , since we will later use it as a reference plane for ionic coordinates of a half-space. The full curve represents schematically the electron density profile $n(r)$ for each particular state. The arrows indicate the order of our steps, and each is labelled with an energy $E$ required to perform the step.

State 1 is the bulk metal. The electron density $n_{-}^{\text {bulk }}(r)$ is not uniform, a fact which has sometimes been neglected in previous work where the small non-uniform contribution to the bulk energy has been taken to imply only a small contribution to the surface energy, leading to erroneous results. Using second-order pseudopotential perturbation theory around the bulk jellium problem, we write the energy of state 1 according to equation (2.5) as

$$
\begin{gathered}
E_{1}=E^{\text {jell }}(\text { bulk })-E_{++}^{\text {jell }}(\text { bulk })+\frac{1}{2} \sum_{i \neq I^{\prime}} v^{\text {direct }}\left(\boldsymbol{R}^{0}(\boldsymbol{l})-\boldsymbol{R}^{0}\left(\boldsymbol{l}^{\prime}\right)\right)+n^{0} \int \Delta V_{\mathrm{ps}}^{\text {bulk }}(\boldsymbol{r}) \mathrm{d}^{3} \boldsymbol{r} \\
+\frac{1}{2} \int \chi^{\text {bulk }}\left(\boldsymbol{r}, \boldsymbol{r}^{\prime}\right) \Delta V_{\mathrm{ps}}^{\text {bulk }}(\boldsymbol{r}) \Delta V_{\mathrm{ps}}^{\text {bulk }}\left(\boldsymbol{r}^{\prime}\right) \mathrm{d}^{3} r \mathrm{~d}^{3} \boldsymbol{r}^{\prime}
\end{gathered}
$$


The terms have the same meaning as in equation (2.5). In particular, $\Delta V_{\mathrm{ps}}^{\text {bulk }}(r)$ is the pseudopotential perturbation due to discrete ions at the perfect bulk lattice positions $\boldsymbol{R}^{0}(l) ; n^{0}$ is the average bulk electron number density.

State 2 has a uniform electron density equal to $n^{0}$. The electron gas is in the manybody state of bulk jellium, despite the presence of the ions. This is not of course the ground state of the pseudo-Hamiltonian $\hat{\dagger} \hat{H}$ but it is a well defined quantum state and its expectation energy $\langle 2|\hat{\jmath}| 2\rangle$ is exactly

$$
\begin{gathered}
E_{2}=E^{\text {jell }}(\text { bulk })-E_{++}^{\text {jell }}(\text { bulk })+n^{0} \int \Delta V_{\mathrm{ps}}^{\text {bulk }}(\boldsymbol{r}) \\
+\frac{1}{2} \sum_{l \neq l^{\prime}} v^{\text {direct }}\left(\boldsymbol{R}^{0}(l)-\boldsymbol{R}^{0}\left(\boldsymbol{l}^{\prime}\right)\right) .
\end{gathered}
$$

We note that (3.2) can be regarded in two ways:

(i) it is the exact expectation energy of state 2 ;

(ii) it is also the energy up to first order of the bulk metal, which is the starting point of Lang and Kohn's first-order surface energy calculation (see figure 5 of their paper).

In state 3 the system has been cleaved and the electron density in each half is $n_{-}^{\text {jell }}(z)$, the density obtained by solving the Kohn-Sham equations with a jellium halfspace background (Lang and Kohn 1970). Note that $n_{-}^{\text {jell }}(z)$ is not relaxed to the ion cores. To specify a definite quantum state we invoke the theorem of Hohenberg and Kohn (1964) which asserts that there is only one ground state of Coulomb-interacting electrons (requiring some external potential) which yields this density. The expectation energy $E_{3}=\langle 3|\dot{x}| 3\rangle$ is given exactly within density functional theory by

$$
\frac{1}{2} E_{3}=E^{\text {jell }}(\operatorname{surf})-E_{++}^{\text {jell }}(\text { surf })+E_{\text {ii }}(\operatorname{surf})+\int n_{-}^{\text {jell }}(z) \Delta V_{\mathrm{ps}}^{\text {surf }}(r) \mathrm{d}^{3} r
$$

where

$$
E_{i i}(\text { surf })=\frac{1}{2} \sum_{\substack{l \neq l \\ l, l_{3}<0}} v^{\text {direct }}\left(\boldsymbol{R}^{0}(l)-\boldsymbol{R}^{0}\left(\boldsymbol{l}^{\prime}\right)\right)
$$

and the other terms are defined as in equation (2.5), with all quantities now referring to a half-space problem. The pseudo-ion positions $\left\{\boldsymbol{R}^{0}(l)\right\}$ are spaced as in the bulk, however (i.e. we omit any ionic layer relaxation or reconstruction at this stage). Equation (3.3) is the exact energy of the artificial state 3 within local density functional theory. It can also be regarded, however, as the energy of the true metallic surface system within first-order pseudopotential perturbation theory: thus it is the energy used by Lang and Kohn (1970) for their final state when calculating their surface energy $\sigma^{\mathrm{LK}}$.

State 4 consists of the two semi-infinite metals with the electron density $n_{-}^{\text {surf }}(r)$ relaxed in three dimensions to accommodate the equally spaced ions. From equation (2.5) the energy of each half-space up to second order in $\Delta V_{\text {surf }}^{\mathrm{ps}}$ is

$$
\begin{gathered}
\frac{1}{2} E_{4}=E^{\text {jell }}(\text { surf })-E_{++}^{\text {jell }}(\operatorname{surf})+E_{\mathrm{ii}}(\operatorname{surf})+\int n_{-}^{\text {jell }}(z) \Delta V_{\mathrm{ps}}^{\text {surf }}(\boldsymbol{r}) \mathrm{d}^{3} \boldsymbol{r} \\
+\frac{1}{2} \int \chi^{\text {surf }}\left(\boldsymbol{r}, \boldsymbol{r}^{\prime}\right) \Delta V_{\mathrm{ps}}^{\text {surf }}(\boldsymbol{r}) \Delta V_{\mathrm{ps}}^{\text {surf }}\left(\boldsymbol{r}^{\prime}\right) \mathrm{d}^{3} r \mathrm{~d}^{3} \boldsymbol{r}^{\prime} .
\end{gathered}
$$

It is now simple to obtain the energy differences indicated in figure 1. From (3.1) and (3.2),

$$
E_{12}=E_{2}-E_{1}=-\frac{1}{2} \int \chi^{\text {bulk }}\left(\boldsymbol{r}, \boldsymbol{r}^{\prime}\right) \Delta V_{\mathrm{ps}}^{\text {bulk }}(\boldsymbol{r}) \Delta V_{\mathrm{ps}}^{\text {bulk }}\left(\boldsymbol{r}^{\prime}\right) \mathrm{d}^{3} r \mathrm{~d}^{3} r^{\prime}
$$

$+\hat{\mathscr{H}}=\int \hat{\psi}^{+}(r) \hat{\psi}(r) V_{\mathrm{ps}}(r) \mathrm{d}^{3} r+\hat{H}_{\mathrm{ellel}}+E_{10 \mathrm{n}-10 \mathrm{n}}$ 
By explicit construction of the states 2 and 3 (see above) they have the initial and final energies of Lang and Kohn's (1970) first-order surface energy calculationt. Thus

$$
E_{23}=E_{3}-E_{2}=2 A \sigma^{\mathrm{LK}} .
$$

From (3.5) and (3.3),

$$
E_{34}=E_{4}-E_{3}=1 \cdot \int \chi^{\text {surf }}\left(\boldsymbol{r}, \boldsymbol{r}^{\prime}\right) \Delta V_{\mathrm{ps}}^{\text {surf }}(\boldsymbol{r}) \Delta V_{\mathrm{ps}}^{\text {surf }}\left(\boldsymbol{r}^{\prime}\right) \mathrm{d}^{3} \boldsymbol{r} \mathrm{d}^{3} \boldsymbol{r}^{\prime} .
$$

The surface energy $\sigma$ is the energy, per unit surface area created, required to go from state 1 to state 4 . Thus up to second order

$\sigma=\left(E_{4}-E_{1}\right) / 2 A=\left(E_{34}+E_{23}+E_{12}\right) / 2 A=\sigma^{\mathrm{LK}}+\left(E_{12}+E_{34}\right) / 2 A$.

Unsurprisingly, our result (3.9) differs from Lang and Kohn's first-order estimate $\sigma^{\mathrm{LK}}$ by two terms (3.8) and (3.6) which are of second order in the pseudopotential perturbation $\Delta V_{\mathrm{ps}}$.

Equation (3.9) is not suitable for numerical calculation as it stands because $E_{12}$ and $E_{34}$ are both bulk terms which almost cancel to yield a surface term. To combine these terms it is convenient once again to introduce the pseudopotential perturbation $\Delta v^{\mathrm{ps}}(\boldsymbol{r})$ due to a single ion at the origin, minus a block of jellium background of equal charge (see equation (2.7)). Then for the case of equally spaced ions we are considering here,

$$
\Delta V_{\mathrm{ps}}^{\text {buik }}(r)=\sum_{l} \Delta v^{\mathrm{ps}}\left(r-\boldsymbol{R}^{0}(l)\right)
$$

and, for the left-hand semi-infinite metal slab,

$$
\Delta V_{\mathrm{ps}}^{\text {surf }}(r)=\sum_{l: l_{3}=-\frac{1}{2},-\frac{3}{2}, \ldots} \Delta v^{\mathrm{ps}}\left(\boldsymbol{r}-\boldsymbol{R}^{0}(l)\right) .
$$

In (3.10) and (3.11) we have placed our origin at the cleavage plane (see broken vertical lines in figure 1) so that the $z$ coordinates of the layers of ions are

$$
R_{3}^{0}(l)=l_{3} d, \quad l_{3}= \pm \frac{1}{2}, \pm \frac{3}{2}, \pm \frac{5}{2} \ldots
$$

for the bulk, with $l_{3}=-\frac{1}{2},-\frac{3}{2},-\frac{5}{2} \ldots$ for the left-hand surface. Using the identity

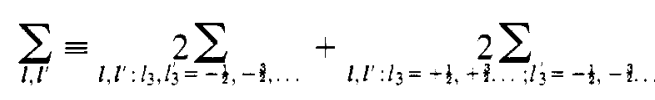

which is valid for summands with inversion symmetry about the origin $\ddagger$, we decompose (3.6) and combine it with (3.8) using (3.10) and (3.11). Then (3.9) becomes

$$
\sigma=\sigma^{\mathrm{LK}}+\sigma_{\mathrm{k} . \mathrm{i}}+\sigma_{\delta x}
$$

where

$$
\begin{aligned}
& \sigma_{\xi, \frac{1}{3}}=-\frac{1}{2 A} \sum_{l: l_{3}=\frac{1}{2}, \ldots, \ldots} \sum_{l^{\prime}: l_{3}=-\frac{1}{2},-\frac{3}{2}, \ldots} \int \mathrm{d}^{3} r \mathrm{~d}^{3} r^{\prime} \chi^{\text {bulk }}\left(r-r^{\prime}\right) \\
& \times \Delta v^{\mathrm{ps}}\left(\boldsymbol{r}-\boldsymbol{R}^{0}(l)\right) \Delta v^{\mathrm{ps}}\left(\boldsymbol{r}^{\prime}-\boldsymbol{R}^{0}\left(\boldsymbol{l}^{\prime}\right)\right)
\end{aligned}
$$

is the negative of the second-order interaction between two half-lattices of ions in the

\footnotetext{
† In obtaining the energy $\sigma^{\mathrm{LK}}$, Lang and Kohn actually used two steps (see their figure 5) whose details are not relevant to our argument.

$\ddagger$ This symmetry exists for all metals and faces we consider, with our choice of origin in the cleavage plane.
} 
bulk, and

$$
\begin{gathered}
\sigma_{\delta \chi}=\frac{1}{2 A} \sum_{l, l^{\prime}: l_{3}, l_{3}=-\frac{1}{2},-\frac{3}{2} \ldots} \int \mathrm{d}^{3} r \mathrm{~d}^{3} \boldsymbol{r}^{\prime}\left(\chi^{\text {surf }}\left(\boldsymbol{r}, \boldsymbol{r}^{\prime}\right)-\chi^{\text {bulk }}\left(\boldsymbol{r}-\boldsymbol{r}^{\prime}\right)\right) \\
\times \Delta v^{\mathrm{ps}}\left(\boldsymbol{r}-\boldsymbol{R}^{0}(l)\right) \Delta v^{\mathrm{ps}}\left(\boldsymbol{r}^{\prime}-\boldsymbol{R}^{0}\left(\boldsymbol{l}^{\prime}\right)\right)
\end{gathered}
$$

represents the difference in second-order energy due to having surface rather than bulk electron response in each semi-infinite surface system.

Numerical evaluation of (3.15) is facilitated by Fourier-transforming all quantities in three dimensions. This gives, for a primitive surface lattice,

$$
\begin{aligned}
\sigma_{\frac{1,1}{2}}=\frac{-1}{4 \pi A_{0}^{2}} \sum_{\substack{l_{3}<0 \\
l_{3}>0}} \int_{-\infty}^{\infty} \mathrm{d} q_{z} \sum_{\boldsymbol{G}} \chi^{\text {bulk }}(|\boldsymbol{g}|) \\
\quad \times \Delta v^{\mathrm{ps}}(-\boldsymbol{g}) \Delta v^{\mathrm{ps}}(\boldsymbol{g}) \exp \left[\mathrm{ig} \cdot\left(\boldsymbol{R}^{0}\left(0,0, l_{3}\right)-\boldsymbol{R}^{0}\left(0,0, l_{3}^{\prime}\right)\right)\right]
\end{aligned}
$$

where $\left\{G_{\|}\right\}$are the reciprocal-lattice vectors of the surface lattice structure, and $g=$ $G_{\|}+q_{z} \hat{z} ; A_{0}$ is the area of the primitive surface direct cell. The sums over $l_{3}$ and $l_{3}^{\prime}$ in (3.17) can be performed analytically as convergent geometric series provided that the $\mathrm{d} q_{z}$ integration is performed as a numerical contour integration above the real axis. Details are given in paper II.

The expression (3.16) only has two-dimensional translational symmetry because of the surface. We therefore Fourier transform all quantities in the surface dimensions using the definition $f\left(\boldsymbol{q}_{\|}, z\right)=\int \mathrm{d}^{2} \boldsymbol{r} \mid f(\boldsymbol{r}) \exp \left(-\mathrm{i} \boldsymbol{q}_{\|} \cdot \boldsymbol{r}\right)$. This yields

$$
\begin{aligned}
\sigma_{\delta \chi}=\frac{1}{2 A^{2}} \sum_{l_{3}, l_{3}<0} & \int_{-\infty}^{\infty} \mathrm{d} z \mathrm{~d} z^{\prime} \sum_{\boldsymbol{G}} \delta \chi\left(\boldsymbol{G}_{\|}, z, z^{\prime}\right) \Delta v^{\mathrm{ps}}\left(-\boldsymbol{G}_{||}, z-R_{3}^{0}(l)\right) \\
& \times \Delta v^{\mathrm{ps}}\left(\boldsymbol{G}_{||}, z^{\prime}-R_{3}^{0}\left(\boldsymbol{l}^{\prime}\right)\right) \exp \left[\boldsymbol{G}_{\|} \cdot\left(\boldsymbol{R}^{0}\left(0,0, l_{3}\right)-R^{0}\left(0,0, l_{3}^{\prime}\right)\right)\right]
\end{aligned}
$$

where $\delta \chi=\chi^{\text {surface }}-\chi^{\text {bulk }}$.

\section{Static relaxation of ionic layer spacings}

We have derived our expressions (3.14)-(3.18) for the surface energy assuming that the ionic layers in each half-space maintain their bulk spacing $d$ when the surface is formed. We now relax the positions of the ionic layers, allowing ions in the $l_{3}$ th layer $\left(l_{3}=-\frac{1}{2}\right.$, $-\frac{3}{2},-\frac{5}{2} \ldots$ ) to change their $z$ coordinate from $R_{3}^{0}(l)=l_{3} d$ to

$$
R_{3}(l)=l_{3} d+\zeta\left(l_{3}\right)=z\left(l_{3}\right)
$$

This layer relaxation is shown schematically in figure 2; see also figure 3 . We do not consider intralayer reconstruction in this section.

The expression (3.5) for the energy $\frac{1}{2} E_{4}$ of a half-space is now modified by replacing $\boldsymbol{R}^{0}(l)$ by $\boldsymbol{R}^{0}(l)+\zeta\left(l_{3}\right) \hat{z}$ in the relevant definitions (3.4) and (3.11). It is convenient once again to introduce compensating blocks of background which fit together to make the jellium background, as was done in $\S 2$. Thus we write (3.11) in the form

$$
\Delta V_{\mathrm{ps}}^{\text {surf }}(\boldsymbol{r})=\sum_{l: l_{3}<0}\left(v^{\mathrm{ps}}(\boldsymbol{r}-\boldsymbol{R}(l))-V_{\text {surf }}^{\mathrm{jell}}(\boldsymbol{r})\right)=\sum_{l: l_{3}<0} \Delta v^{\mathrm{ps}}(r, \boldsymbol{R}(l))
$$

where the compensating block is still centred at $\boldsymbol{R}^{0}(l)$ even though the ion has relaxed to position $\boldsymbol{R}(l)$. Applying a surface Fourier transformation to (3.5) and using (4.2) we 


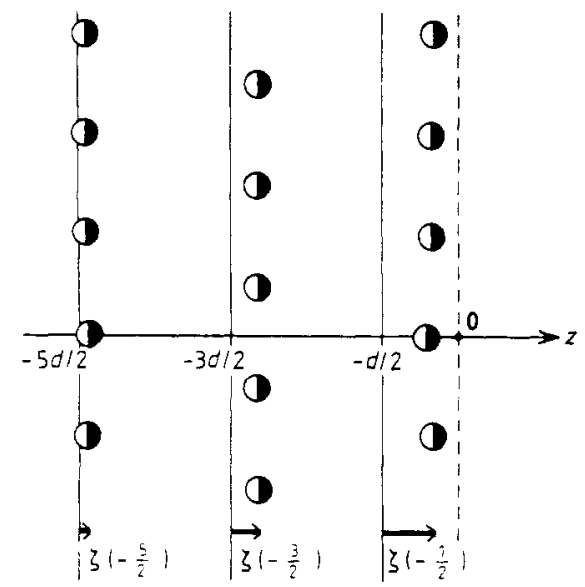

Figure 2. Relaxation of ionic layer positions due to the surface. Note that the 'origin' ion in the $l_{3}$ th plane $\left(l_{3}=-\frac{1}{2},-\frac{3}{2},-\frac{3}{2}, \ldots\right)$ has a position vector $R\left(0,0, l_{3}\right)$ whose $x$ and $y$ components are not in general zero (i.e. successive planes appear relatively displaced when viewed from the vacuum along the $-z$ axis).

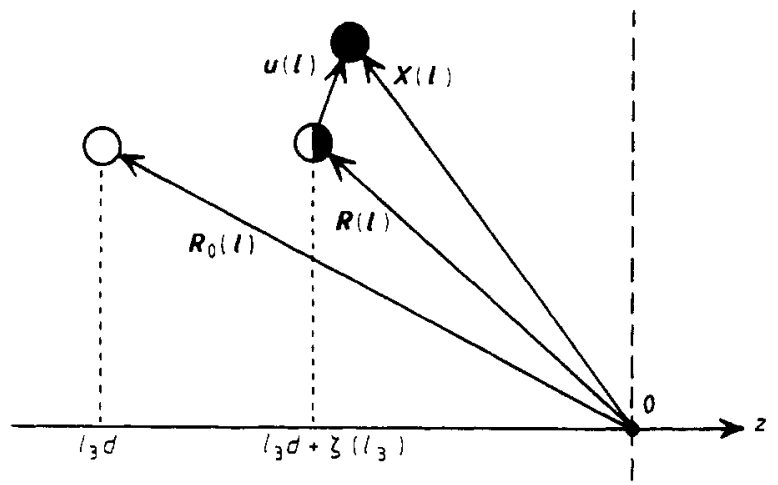

Figure 3. Unrelaxed position $\boldsymbol{R}^{0}(l)$, statically relaxed position $\boldsymbol{R}(l)$ and instantaneous nonequilibrium position $\boldsymbol{X}(l)$ are shown for the $l$ th ion. $\zeta\left(l_{3}\right) z$ is the relaxation of the $l_{3}$ th plane of ions. $u(l)$ is the dynamic excursion of the $l$ th ion.

find the energy of the half-space with displaced layers is

$$
\begin{aligned}
& E\left(\left\{z\left(l_{3}\right)\right\}\right)=U_{0}+\frac{A}{2 A_{0}^{2}} \sum_{l_{3}, l_{3}<0} \sum_{b_{\sharp} \neq 0} \frac{2 \pi Z^{2} e^{2}}{G_{\mid}} \exp \left[-G_{\|}\left|z\left(l_{3}\right)-z\left(l_{3}^{\prime}\right)\right|\right] \\
& l_{3} \neq l_{3} \\
& \times \exp \left[\mathrm{i} \boldsymbol{G}_{\|} \cdot\left(\boldsymbol{R}\left(0,0, l_{3}\right)-\boldsymbol{R}\left(0,0, l_{3}^{\prime}\right)\right)\right] \\
& +\frac{A}{A_{\mathrm{n}}} \sum_{k_{3}<0} \int_{-\infty}^{\infty} \mathrm{d} z n_{-}^{\mathrm{jell}}(z) \Delta v^{\mathrm{ps}}\left(\boldsymbol{G}_{\|}=\mathbf{0}, z, z\left(l_{3}\right)\right) \\
& +\frac{A}{2 A^{2}} \sum_{l_{3}, l_{3}<0} \sum_{G} \int \mathrm{d} z \mathrm{~d} z^{\prime} \chi^{\text {surf }}\left(\boldsymbol{G}_{\mid ;}, z, z^{\prime}\right) \\
& \times \exp \left[\mathrm{i} \boldsymbol{G}_{\|} \cdot\left(\boldsymbol{R}\left(0,0, l_{3}\right)-\boldsymbol{R}\left(0,0, l_{3}^{\prime}\right)\right)\right] \\
& \times \Delta v^{\mathrm{ps}}\left(-\boldsymbol{G}_{\|}, \boldsymbol{z}, z\left(l_{3}\right)\right) \Delta v^{\mathrm{ps}}\left(\boldsymbol{G}_{\|}, z^{\prime}, z\left(l_{3}^{\prime}\right)\right) .
\end{aligned}
$$


Here the constant $U_{0}$ does not depend on the $\left\{z\left(l_{3}\right)\right\}$ : it contains jellium terms and the Coulomb interaction of layers with themselves. $Z e$ is the ionic core charge. The surface lattice is assumed primitive.

To obtain the equilibrium layer positions $\left\{z\left(l_{3}\right)\right\}$ we demand that the 'Kanzaki force' $F(l)$ on each layer $l$ be zero:

$$
F(l) \equiv-\frac{1}{A} \frac{\partial E}{\partial z(l)}=0, \quad l=-\frac{1}{2},-\frac{3}{2},-\frac{5}{2} \ldots
$$

Differentiating (4.3) we obtain

$$
\begin{aligned}
F(l)=\frac{1}{A_{0}} \int_{-\infty}^{\infty} & n_{-}^{\text {jell }}(z) \frac{\partial}{\partial z} v^{\mathrm{ps}}\left(\boldsymbol{G}_{\mid}=\mathbf{0}, z-z(l)\right) \mathrm{d} z \\
& +\frac{1}{A^{2}} \sum_{\boldsymbol{G}_{\|}} \sum_{l_{3}<0}\left[\operatorname{exp~} \boldsymbol{G}_{\|} \cdot\left(\boldsymbol{R}(0,0, l)-\boldsymbol{R}\left(0,0, l_{3}\right)\right)\right] \\
& \times\left(2 \pi Z^{2} e^{2} \operatorname{sgn}\left(z(l)-z\left(l_{3}\right)\right) \exp \left(-G_{\|}\left|z(l)-z\left(l_{3}\right)\right|\right)\right. \\
& \times\left(1-\delta_{l l_{3}}\right)\left(1-\delta_{G_{0} 0}\right)+\iint_{-\infty}^{\infty} \mathrm{d} z \mathrm{~d} z^{\prime} \bar{\chi}^{\text {surf }}\left(G_{\|}, z, z^{\prime}\right) \\
& \left.\times \Delta v^{\mathrm{ps}}\left(-G_{\|}, z, z\left(l_{3}\right)\right) \frac{\partial}{\partial z^{\prime}} v^{\mathrm{ps}}\left(\boldsymbol{G}_{\mid}, z^{\prime}-z(l)\right)\right),
\end{aligned}
$$

where

$$
\bar{\chi}\left(G_{\mid ;}, z, z^{\prime}\right)=\frac{1}{2}\left(\chi\left(G_{\|}, z, z^{\prime}\right)+\chi\left(G_{\|}, z^{\prime}, z\right)\right)
$$

Note that, even though $\chi^{0}\left(\boldsymbol{k}_{\mid}, z, z^{\prime}\right)=\chi^{0}\left(\boldsymbol{k}_{\|}, z^{\prime}, z\right)$, the inhomogeneous screening causes the corresponding screened susceptibilities to be unequal.

Evaluating (4.5) is a computational task of the same order as obtaining the surface energy from (3.18). If we wish to relax only a few layers it is possible to solve the nonlinear equations (4.4) iteratively, using (4.5) at each step. A similar procedure was carried out by Landman et al (1980) who used only the first two terms of (4.5): that is, they neglected screening of the ions and the associated electron-gas compressional kinetic energy effects which we include by the $\chi^{\text {surface }}$ term in (4.5). These screening effects substantially weaken the Coulomb interaction (Perdew and Monnier 1980). Landman et al obtained a large $(\xi / d \approx 20 \%)$ relaxation of the first layer at the (110) face of $\mathrm{Al}$, but for the most densely packed faces of $\mathrm{Al}$ and $\mathrm{Na}$ even the first layer only relaxed by $1 \%$. We feel the proper inclusion of screening, as in equation (4.5), will reduce the relaxations further and we propose that a linearised lattice statics relaxation scheme will be sufficient for all layers except possibly the first. Thus we Taylor-expand the energy (4.3) to second order in the layer displacements $\zeta(l)$ :

$$
\frac{1}{A} E(\{z(l)\}) \simeq \frac{1}{A} E\left(\left\{R_{3}^{0}(l)\right\}\right)-\sum_{l<0} F^{0}(l) \zeta(l)+\frac{1}{2} \sum_{l, l^{\prime}<0} D\left(l, l^{\prime}\right) \zeta(l) \zeta\left(l^{\prime}\right)
$$

where $F^{0}(l)$ is evaluated at the bulk spacing (i.e. $z(l)=R_{3}^{0}(l)$ ) and the one-dimensional 
stiffness matrix $D$ is obtained by differentiating (4.5):

$$
\begin{aligned}
& D\left(l, l^{\prime}\right)=-\frac{\delta_{l l^{\prime}}}{A_{0}} \int_{-\infty}^{\infty} n_{-}^{\text {jell }}(z) \frac{\partial^{2}}{\partial z^{2}} v^{\mathrm{ps}}\left(\boldsymbol{G}_{\|}=0, z-z(l)\right) \mathrm{d} z \\
&+ \frac{1}{A_{0}^{2}} \sum_{G} \sum_{l_{3}<0} \cos \left[\boldsymbol{G}_{\mid} \cdot\left(\boldsymbol{R}(0,0, l)-\boldsymbol{R}\left(0,0, l_{3}\right)\right)\right] \\
& \times\left[\left(\delta_{l l^{\prime}}-\delta_{l l^{\prime}}\right) \boldsymbol{G}_{\|} \exp \left(-G_{\|}\left|z(l)-z\left(l_{3}\right)\right|\right) 2 \pi z^{2} e^{2}+\iint_{-\infty}^{\infty}\right. \\
& \times \mathrm{d} z \mathrm{~d} z^{\prime} \chi^{\text {-surf }}\left(\boldsymbol{G}_{\|}, z, z^{\prime}\right)\left(\delta_{l l^{\prime}} \frac{\partial^{2}}{\partial z^{2}} v^{\mathrm{ps}}\left(-\boldsymbol{G}_{\|}, z-z(l)\right) \cdot \Delta v^{\mathrm{ps}}\left(\boldsymbol{G}_{\|}, z^{\prime}-z\left(l_{3}\right)\right)\right. \\
&\left.\left.+\delta_{l_{3} l^{\prime}} \frac{\partial}{\partial z} v^{\mathrm{ps}}\left(-\boldsymbol{G}_{\|}, z-z(l)\right) \frac{\partial}{\partial z^{\prime}} v^{\mathrm{ps}}\left(\boldsymbol{G}_{\|}, z^{\prime}-z\left(l_{3}\right)\right)\right)\right] .
\end{aligned}
$$

To determine the layer displacements we minimise (4.6) with respect to the $\{\xi(l)\}$. This is solved by a matrix inversion

$$
\zeta(l)=\left(D^{-1}\right)_{l l} F^{0}\left(l^{\prime}\right)
$$

and the resultant lowering of energy is

$$
\Delta E \text { (layer) }=-\frac{1}{2} \sum_{l} F^{0}(l) \zeta(l) .
$$

Thus if we choose to allow relaxation of the first $M$ layers of the metal surface, we have an $M \times M$ matrix inversion to perform. Note that if we relaxed all layers our matrix $D$ would be singular since an equal displacement of all layers does not change the energy. This well known difficulty of lattice statics is not encountered here as the system is stabilised by layers $M+1, M+2, \ldots$ which are not relaxed.

When relaxing multiple layers as above it is important to work with a correctly chosen pseudopotential $v^{\mathrm{ps}}$ such that the true density is obtained for the bulk metal at zero pressure, as determined from equation (2.5). If this is not done, we expect the layer relaxations $\zeta(l)$ from equation (4.8) to increase as the number of relaxed layers, $M$, increases. This is due to the system relaxing toward bulk density. Even if this occurs, however, we might hope the differences $\zeta(l)-\zeta(l+1)$ will correctly represent true surface effects for the first few layers.

We should mention the approximate calculations of $\mathrm{Al}$ surface layer relaxation by Perdew and Monnier (1980) which agree with some measurements. As they note, however, their method is not quantitatively reliable for the less densely packed faces (see also Perdew 1982). We expect ours to be a substantial improvement.

\section{Harmonic lattice dynamics}

Assuming that we have found the relaxed static equilibrium positions $R(l)=$ $\boldsymbol{R}^{0}(l)+\xi\left(l_{3}\right) \hat{z}$ for the metal surface problem, we can use the energy expression $(2.5)$ to investigate harmonic motion of the lattice in the Born-Oppenheimer approximation. Writing the instantaneous positions of the ions (see figure 3 ) as

$$
X(l)=R(l)+u(l, t)
$$

we obtain the harmonic equations of motion in the usual fashion by expanding (2.5) to 
second order in the displacements $\boldsymbol{u}$ :

$$
M \frac{\mathrm{d}^{2}}{\mathrm{~d} t^{2}} u_{\alpha}(l, t)=-\sum_{l^{\prime}, \beta} \frac{\partial^{2} E}{\partial X_{\alpha}(l) \partial X_{\beta}\left(l^{\prime}\right)} u_{\beta}\left(l^{\prime}, t\right) .
$$

Here $u_{\alpha}$ denotes the $\alpha$ th Cartesian component of displacement and $M$ is the ionic mass. The derivatives of $E$ are to be evaluated at the equilibrium positions $X(l)=R(l)$. Assuming that the bulk crystal structure is preserved in the surface directions $(\alpha=1,2)$, we can solve (5.2) with the ansatz

$$
u_{\alpha}(l, t)=u_{\alpha}\left(\boldsymbol{q}_{1}, l_{3}\right) \exp \left(\mathrm{i} \boldsymbol{q} \cdot \boldsymbol{R}^{0}(l)\right) \exp (-\mathrm{i} \omega t)
$$

thus obtaining (Maradudin et al 1971, Trullinger et al 1973)

$$
\omega^{2} u_{\alpha}\left(\boldsymbol{q}_{\|}, l_{3}\right)=\sum_{l_{3} \beta} D_{\alpha \beta}\left(\boldsymbol{q}_{\mid}, l_{3}, l_{3}^{\prime}\right) u_{\beta}\left(\boldsymbol{q}_{\|}, l_{3}^{\prime}\right)
$$

where the dynamical matrix $D$, for a primitive surface lattice, is

$$
\begin{aligned}
D_{\alpha \beta}\left(\boldsymbol{q} \|, l_{3}, l_{3}^{\prime}\right)= & \frac{1}{M} \sum_{l_{1} l_{2}=-\infty}^{\infty} \exp \left[-\mathrm{i} \boldsymbol{q}_{!} \cdot\left(\boldsymbol{R}\left(l_{1} l_{2} l_{3}\right)-\boldsymbol{R}\left(00 l_{3}^{\prime}\right)\right)\right] \\
& \times \frac{\partial^{2} E}{\partial X_{\alpha}\left(l_{1} l_{2} l_{3}\right) \partial X_{\beta}\left(0,0, l_{3}^{\prime}\right)} .
\end{aligned}
$$

Equation (5.5) is to be evaluated at $\boldsymbol{X}(l)=\boldsymbol{R}(l)$.

Inserting (5.5) into the expression (2.8) for the energy variation in terms of effective one- and two-ion potentials, and Fourier-expanding in the surface direction, we obtain

$$
D_{\alpha \beta}\left(\boldsymbol{q}_{\|}, l_{3}, l_{3}^{\prime}\right)=D_{\alpha \beta}^{1 \text {-body }}\left(\boldsymbol{q}_{\|}, l_{3}, l_{3}^{\prime}\right)+D_{\alpha \beta}^{2-\text { body }}\left(\boldsymbol{q}, l_{3}, l_{3}^{\prime}\right)
$$

where

$$
D_{\alpha \beta}^{1 \text {-body }}\left(\boldsymbol{q}_{\|}, l_{3}, l_{3}^{\prime}\right)=\frac{1}{M} \delta_{\alpha 3} \delta_{\beta 3} \delta_{l_{3} l_{3}^{\prime}{ }_{-\infty}} \int_{-\infty}^{\text {jell }}(z) \frac{\partial^{2}}{\partial z^{2}} v^{\mathrm{ps}}\left(\boldsymbol{k}_{\|}=\mathbf{0}, z-\boldsymbol{R}_{3}\left(l_{3}\right)\right) \mathrm{d} z
$$

and

$$
\begin{aligned}
D_{\alpha \beta}^{2-\text { body }}\left(\boldsymbol{q}_{\|}, l_{3}, l_{3}^{\prime}\right) & =\frac{1}{M A_{0}} \sum_{G} \sum_{l_{3}^{\prime \prime}<0} \exp \left[\mathrm{i} \boldsymbol{G}_{\mid} \cdot\left(\boldsymbol{R}\left(0,0, l_{3}\right)-\boldsymbol{R}\left(0,0, l_{3}^{\prime}\right)\right)\right] \\
\times & {\left[\delta_{l_{3}, l_{3}} \varphi_{(\alpha: \beta)}^{\mathrm{ccr}}\left(\boldsymbol{q}+\boldsymbol{G}_{\|}, R_{3}\left(l_{3}\right), R_{3}\left(l_{3}^{\prime}\right)\right)-\delta_{l_{3}, l_{3}} \varphi_{(\alpha \beta)}^{\mathrm{sc}}\left(\boldsymbol{G}_{\|,}, R_{3}\left(l_{3}\right), R_{3}\left(l_{3}^{\prime \prime}\right)\right)\right] . }
\end{aligned}
$$

Here the derivatives $\varphi_{(\alpha: \beta)}^{\mathrm{scr}}$ and $\varphi_{(\alpha \beta)}^{\mathrm{scr}}$ of the two-dimensionally Fourier-transformed effective ion-ion potential can be expressed so that the derivatives appear explicitly only on the pseudopotential $v^{\mathrm{ps}}$ and on the Coulomb interaction $v^{\text {direct }}$, both of which are usually known analytically:

$$
\begin{gathered}
\varphi_{(\alpha: \beta)}^{\mathrm{scr}}\left(\boldsymbol{k}_{\|}, X_{3}, X_{3}^{\prime}\right)=-v_{(\alpha \beta)}^{\mathrm{direct}}\left(\boldsymbol{k}_{i}, X_{3}-X_{3}^{\prime}\right)+\int_{-\infty}^{\infty} \mathrm{d} z \mathrm{~d} z^{\prime} \bar{\chi}^{\text {surt }}\left(\boldsymbol{k}_{i,}, z, z^{\prime}\right) \\
\times v_{(\alpha)}^{\mathrm{ps}}\left(-\boldsymbol{k}_{i}, z-X_{3}\right) v \mathrm{z}_{\beta}^{\mathrm{ps}}\left(\boldsymbol{k}_{\mathrm{i}}, z^{\prime}-X_{3}^{\prime}\right)
\end{gathered}
$$




$$
\begin{gathered}
\varphi_{(\alpha \beta:)}^{\mathrm{scr}}\left(\boldsymbol{k}_{\|}, X_{3}, X_{3}^{\prime}\right)=-v_{(\alpha \beta)}^{\mathrm{direct}}\left(\boldsymbol{k}_{\mid ;}, X_{3}-X_{3}^{\prime}\right)-\int_{-\infty}^{\infty} \mathrm{d} z \mathrm{~d} z^{\prime} \bar{\chi}^{\text {surf }}\left(\boldsymbol{k}_{\|}, z, z^{\prime}\right) \\
\times v_{(\alpha \beta)}^{\mathrm{ps}}\left(-\boldsymbol{k}_{\mid}, z-X_{3}\right) \Delta v^{\mathrm{ps}}\left(\boldsymbol{k}_{\mid}, z^{\prime}-X_{3}^{\prime}\right) .
\end{gathered}
$$

In (5.9) and (5.10) we have used the abbreviations

$$
\begin{aligned}
& f_{(\alpha)}\left(\boldsymbol{k}_{\mid}, z\right)= \begin{cases}\mathrm{i} k_{\| \alpha} f\left(\boldsymbol{k}_{\|}, z\right) \\
\partial f\left(\boldsymbol{k}_{\|}, z\right) / \partial z\end{cases} \\
& f_{(\alpha \beta)}\left(\boldsymbol{k}_{\mid}, z\right)=\left(f_{(\alpha)}\right)_{(\beta)}\left(\boldsymbol{k}_{\mid,}, z\right)
\end{aligned}
$$

for an arbitrary function $f\left(\boldsymbol{k}_{\|}, \boldsymbol{z}\right)$ in two-dimensional Fourier space. Also, $\bar{\chi}^{\text {surf }}$ is the symmetrised screened susceptibility defined in equation (4.5a).

By comparing (5.8)-(5.10) with (3.18) we find that evaluation of any one element of the dynamical matrix is a computational task similar in magnitude to that of evaluating the surface energy. (The sum over $l_{3}^{\prime \prime}$ in (5.8) is rapidly convergent except for $G=\mathbf{0}$.)

\section{Temperature-dependent surface free energy: Einstein and self-consistent Einstein models}

A major goal of this development is to estimate the temperature-dependent lattice dynamical contribution to the surface free energy of solid metals. We recall that the most reliable surface energy determinations come from liquid surface tension measurements (Tyson and Miller 1977). It is necessary to extrapolate though freezing and down to $T=0 \mathrm{~K}$, in order to make contact with existing microscopic surface energy calculations (Wawra 1975a, b). We concern ourselves here with the solid phase extrapolation.

In principle it is possible to obtain integrated quantities such as the surface vibrational free energy by calculating the frequencies from the dynamical matrix (5.7), (5.8), then performing a suitable layer summation and surface zone integration (see, e.g., Maradudin et al 1971, Allen and de Wette 1969). This is a formidable task, however, because of the complicated form of the effective ionic potentials (2.9), (2.10).

Furthermore, such an approach only yields results in the strictly harmonic regime. In the face of these difficulties we resort to simpler lattice dynamical schemes, the Einstein and self-consistent Einstein models. The latter is capable of describing a degree of anharmonicity which is an advantage at temperatures near to, but below, the melting temperature.

\subsection{Non-self-consistent Einstein model}

The 'standard' or 'non-self-consistent' Einstein model postulates that each ion (labelled $l$ ) moves in its own independent potential $V_{l}^{E}$ which is harmonic in the excursion $u(l)$ :

$$
V_{l}^{E}(\boldsymbol{u}(\boldsymbol{l}))=\frac{1}{2} M \sum_{\alpha, \beta=1}^{3}\left(\omega^{2}\right)_{\alpha \beta} u_{\alpha}(l) u_{\beta}(l) .
$$

Here the Einstein frequency matrix $\omega$ is obtained from an expansion of the total energy $E$ (equation (2.8)) up to second order in the excursion $\boldsymbol{u}(l) \equiv \boldsymbol{X}(\boldsymbol{l})-\boldsymbol{R}(l)$, holding 
all other ionic positions $\left\{\boldsymbol{X}\left(\boldsymbol{l}^{\prime}\right), \boldsymbol{l}^{\prime} \neq \boldsymbol{l}\right\}$ constant at their equilibrium values $\left\{\boldsymbol{R}\left(\boldsymbol{l}^{\prime}\right)\right\}$ :

$$
\frac{1}{2} M\left(\omega^{2}(l)\right)_{\alpha \beta}=\left.\frac{\partial^{2} E}{\partial X_{\alpha}(l) \partial X_{\beta}(l)}\right|_{\boldsymbol{X}\left(l^{\prime}\right)=\boldsymbol{R}\left(l^{\prime}\right)} .
$$

Putting (6.2) into (2.8) and Fourier-expanding all quantities in the directions parallel to the surface we obtain one-ion and ion-ion contributions:

$$
\begin{aligned}
& \omega^{2}(l)=\left(\omega^{2}(l)\right)^{\text {ion }}+\left(\omega^{2}(l)\right)^{\text {ion-ion }} \\
& \frac{1}{2} M\left(\omega^{2}(l)\right)_{\alpha \beta}^{\text {ion }}=\delta_{\alpha \beta} \delta_{\beta 3} \int_{-\infty}^{\infty} \mathrm{d} z n_{-}^{\text {jell }}(z) \frac{\partial^{2} v^{\text {ps }}}{\partial z^{2}}\left(\boldsymbol{k}_{\mid !}=\mathbf{0}, z-R_{3}(l)\right) \\
& \frac{1}{2} M\left(\omega^{2}(l)\right)_{\alpha \beta}^{\mathrm{ion}-\mathrm{ion}}=\frac{1}{(2 \pi)^{2}} \int_{\text {all } k} \varphi_{(\alpha ; \beta)}^{\mathrm{scr}}\left(\boldsymbol{k}_{||,}, R_{3}(l), R_{3}(l)\right) \mathrm{d}^{2} k_{\mid} \\
& -\frac{1}{A_{0}} \sum_{\boldsymbol{G}_{\mid}} \sum_{\boldsymbol{l}^{\prime}} \exp \left[\mathrm{i} \boldsymbol{G}_{\|} \cdot\left(\boldsymbol{R}^{0}(\boldsymbol{l})-\boldsymbol{R}^{0}\left(\boldsymbol{l}^{\prime \prime}\right)\right)\right] \\
& \times \varphi_{(\alpha \beta)}^{\mathrm{scr}}\left(G_{\| !}, R_{3}\left(l_{1} l_{2} l_{3}\right), R_{3}\left(l_{1} l_{2} l_{3}^{\prime}\right)\right) .
\end{aligned}
$$

Here the notation is as in equations (5.8)-(5.12).

Each symmetric frequency matrix $\omega^{2}(l)$ has three real eigenvalues $\omega_{\mu}^{2}(l), \mu=1,2,3$, characterising decoupled vibrations in three orthogonal directions. The vibrational contribution to the crystal free energy is the sum of harmonic oscillator free energies, three for each ion (see Dobson and Ashcroft 1977):

$$
F^{\mathrm{vib}}=\sum_{l} \sum_{\mu=1}^{3} k_{\mathrm{B}} T \ln \left[2 \sinh \left(\omega_{\mu}(l) / 2 k_{\mathrm{B}} T\right)\right] .
$$

Equations (6.3) are written in a mathematical form suitable for metal surfaces, with crystal translational invariance broken in the $z$ or ' 3 ' direction. Nevertheless for purposes of comparison we can also use (6.3) to express the bulk Einstein frequencies $\omega_{\mu}(\mathrm{bulk})$, provided that $n_{-}^{\text {jell }}$ in $(6.3 b)$ is interpreted as the zeroth-order uniform bulk electron density instead of the Lang-Kohn density, while $\varphi^{\text {scr }}$ appearing in $(6.3 c)$ is defined as in (5.9), (5.10), but with the bulk susceptiblity $\chi^{\text {bulk }}$ in place of $\chi^{\text {surf }}$.

The vibrational contribution to the surface free energy (per unit surface area formed) is

$$
\begin{aligned}
\sigma^{\mathrm{vib}}=\frac{1}{2 A}\left(2 F^{\mathrm{vib}}\left(\frac{1}{2} \text {-space }\right)-F^{\mathrm{vib}}(\text { bulk })\right) \\
=\frac{k_{\mathrm{B}} T}{A_{0}} \sum_{l_{3}<0} \sum_{\mu=1}\left\{\ln \left[2 \sinh \left(\omega_{\mu}\left(0,0, l_{3}\right) / 2 k_{\mathrm{B}} T\right)\right]\right. \\
\left.-\ln \left[2 \sinh \left(\omega_{\mu}(\text { bulk }) / 2 k_{\mathrm{B}} T\right)\right]\right\}
\end{aligned}
$$

where $A_{0}$ is the primitive direct surface cell area.

In practice we expect only the first layer or two into the surface to have Einstein frequencies very different from the bulk. In particular we expect the surface layer to have a somewhat altered frequency $\omega_{3}$ for motion perpendicular to the surface. As a first approximation, then, only one or two terms in the sum (6.5) will be large. Smaller contributions from deeper layers, if significant, can be treated linearly in the difference $\Delta\left(\omega^{2}\right)$ between frequency matrices (6.3) for surface and bulk systems. Like the static 
surface energy (3.14), layer sums over such differences can presumably be expressed usefully in terms of a ' $\frac{1}{2}-\frac{1}{2}$ ' bulk contribution and a ' $\delta \chi$ ' part due to surface effects on the electronic screening.

\subsection{Self-consistent Einstein model}

The scheme just described does not allow for anharmonic effects at high temperature. Such effects can be partly included by variational schemes of the self-consistent phonon type. The simplest variational scheme of this type is the self-consistent Einstein model, which is well suited to situations of broken crystal symmetry such as surfaces: see Matsubara and Kamiya (1977) and Dobson and Ashcroft (1977). It gives a rigorous upper bound on the free energy of a lattice dynamical system, and reduces to the standard Einstein model in the limit of small excursions.

We choose a reference Hamiltonian $\mathscr{H}_{0}$ describing a fictitious set of independent, possibly anisotropic, harmonic oscillators:

$$
\hat{\mathscr{H}}_{0}=\frac{1}{2} \sum_{l}\left(\hat{p}(l)^{2} / M+M u(l) \cdot \omega^{2}(l) \cdot u(l)\right)
$$

where $\boldsymbol{u}(l)$ is the excursion of the $l$ th lattice ion about its mean position $R(l)$. We consider $\{\boldsymbol{R}(l)\}$ and the frequency matrices $\{\omega(l)\}$ to be a set of variational parameters chosen to minimise a trial free energy, and capable of describing temperature-dependent changes in layer relaxation and thermal motion. This approach differs from the non-self-consistent Einstein model because, in the latter, $\omega^{2}$ is fixed as a second derivative calculated at static equilibrium.

We use (6.6) in conjunction with the Gibbs-Bogoliubov variational principle for the exact Helmholtz free energy $F$. This states that

$$
\begin{aligned}
F \leqslant F_{\text {trial }} \equiv F_{0} & +\operatorname{Tr}\left[\exp \left(-\beta \hat{\mathscr{H}}_{0}\right)\left(\hat{V}-\hat{V}_{0}\right)\right] / \operatorname{Tr}\left[\exp \left(-\beta \hat{\mathscr{H}}_{0}\right)\right] \\
& \equiv F_{0}+\left\langle\hat{V}-\hat{V}_{0}\right\rangle_{0} .
\end{aligned}
$$

A proof of this result is given in Feynman (1972), p. 67. In (6.7),

$$
F_{0}=-k_{\mathrm{B}} T \ln \operatorname{Tr} \exp \left(-\beta \mathscr{H}_{0}\right)
$$

is the exact free energy corresponding to the fictitious Hamiltonian (6.6) at temperature $T=\left(k_{\mathrm{B}} \beta\right)^{-1} ; \hat{V}_{0}$ is the potential part of $(6.6)$ and $\hat{V}$ is the true lattice potential energy which we take as the linear response expression $(2.8)-(2.10)$ :

$$
\hat{V}(\{X(I)\}) \equiv E(\{X(I)\})
$$

Because (6.6) describes decoupled harmonic oscillators, the traces prescribed in (6.7) are easily performed quantally at arbitrary temperature (Messiah 1965):

$$
\begin{aligned}
& F_{0}=k_{\mathrm{B}} T \sum_{l} \operatorname{Tr} \ln \left[2 \sin \left(\frac{1}{2} \hbar \beta \omega(l)\right)\right] \\
& \left\langle-\hat{V}_{0}\right\rangle_{0}=-\sum_{l} \operatorname{Tr}\left[\frac{1}{4} \hbar \omega(l) \operatorname{coth}\left(\frac{1}{2} \beta \hbar \omega(l)\right)\right] .
\end{aligned}
$$

The expectation value of the true potential $\hat{V}$ is

$$
\langle\hat{V}\rangle_{0}=E_{0}+\sum_{l} \tilde{w}(\boldsymbol{R}(l))+\frac{1}{2} \sum_{l, l^{\prime}} \tilde{\varphi}^{\mathrm{scr}}\left(\boldsymbol{R}(l), \boldsymbol{R}\left(\boldsymbol{l}^{\prime}\right)\right)
$$

where the thermally smeared ion and ion-ion potentials $\tilde{w}$ and $\varphi^{\text {scr }}$ can be written so that 
the smearing only enters via modification of the bare pseudopotential and direct ion interaction:

$$
\begin{aligned}
& \tilde{w}(\boldsymbol{R}(l))=\int n_{-}^{\mathrm{jell}}(\boldsymbol{r}) \tilde{\Delta} v^{\mathrm{ps}}(\boldsymbol{r}, \boldsymbol{R}(\boldsymbol{l})), \\
& \tilde{\varphi}^{\mathrm{scr}}\left(\boldsymbol{R}(\boldsymbol{l}), \boldsymbol{R}\left(\boldsymbol{l}^{\prime}\right)\right)=\int \mathrm{d}^{3} r \mathrm{~d}^{3} r^{\prime} \chi\left(\boldsymbol{r}, \boldsymbol{r}^{\prime}\right) \tilde{\Delta} v^{\mathrm{ps}}(\boldsymbol{r}, \boldsymbol{R}(\boldsymbol{l})) \tilde{\Delta} v^{\mathrm{ps}}\left(\boldsymbol{r}^{\prime}, \boldsymbol{R}\left(\boldsymbol{l}^{\prime}\right)\right) \\
& +\tilde{v}^{\mathrm{direct}}\left(\boldsymbol{R}(\boldsymbol{l})-\boldsymbol{R}\left(\boldsymbol{l}^{\prime}\right)\right) .
\end{aligned}
$$

Here,

$$
\begin{aligned}
\tilde{\Delta} v^{\mathrm{ps}}(\boldsymbol{r}, \boldsymbol{R}(l))= & \frac{1}{(2 \pi)^{3}} \int \mathrm{d}^{3} k v^{\mathrm{ps}}(k) \exp \left[-\frac{1}{2} k \cdot \gamma(l) \cdot k\right] \exp [\mathrm{i} \boldsymbol{k} \cdot(\boldsymbol{r}-\boldsymbol{R}(l))] \\
& -v^{\text {block }}\left(\boldsymbol{r}-\boldsymbol{R}^{0}(l)\right)
\end{aligned}
$$

is the potential due to a thermally smeared pseudo-ion, minus a block of positive background, and

$$
\begin{aligned}
& \tilde{v}^{\text {direct }}(\boldsymbol{R}(l)-\left.\boldsymbol{R}\left(\boldsymbol{l}^{\prime}\right)\right)=\frac{1}{(2 \pi)^{3}} \int \mathrm{d}^{3} k v^{\text {direct }}(k) \exp \left[-\frac{1}{2} \boldsymbol{k} \cdot\left(\gamma(l)+\gamma\left(\boldsymbol{l}^{\prime}\right)\right) \cdot \boldsymbol{k}\right] \\
& \times \exp \left[\mathrm{i} \boldsymbol{k} \cdot\left(\boldsymbol{R}(\boldsymbol{l})-\boldsymbol{R}\left(\boldsymbol{l}^{\prime}\right)\right)\right]
\end{aligned}
$$

is the thermally smeared direct ion-ion potential.

In (6.15) and (6.16), the mean-square excursion matrix $\gamma$ has elements $\gamma_{\alpha \beta}(l)=$ $\left\langle u_{\alpha}(l) u_{\beta}(l)\right\rangle$ and can be evaluated as follows:

$$
\gamma(l)=(\hbar / 2 m) \omega(l)^{-1} \operatorname{coth}\left(\frac{1}{2} \beta \hbar \omega(l)\right)
$$

(In (6.10)-(6.17), a function $f(\omega)$ of a matrix is defined as a matrix with the same eigenvectors as $\omega$ but with eigenvalues $\left\{f\left(\omega_{\mu}\right), \mu=1,2,3\right\}$. Thus in particular $\operatorname{Tr} f(\omega)=\Sigma_{\mu=1}^{3} f\left(\omega_{\mu}\right)$.) If $v^{\text {direct }}$ is the Coulomb potential, isotropic thermal smearing has essentially no effect on $v^{\text {direct }}\left(\boldsymbol{R}-\boldsymbol{R}^{\prime}\right)$ when $\left|\boldsymbol{R}-\boldsymbol{R}^{\prime}\right| \geq u_{\mathrm{RMS}}$, because of Gauss's theorem. For anisotropic smearing, however, multipole terms are present in $\tilde{v}^{\text {direct }}$.

In principle one could obtain the optimal bound on the vibrational free energy directly from (6.7) by varying $\{\omega(l)\}$ and $\{\boldsymbol{R}(l)\}$ numerically until the trial free energy (the sum of $(6.10),(6.11)$ and $(6.12)$ ) is minimised. In practice it may be preferable to obtain the frequencies from an equation similar to (6.3). By taking the derivative of (6.10)-(6.17) with respect to the elements of $\omega(l)$, one can formally obtain the minimisation equation for the trial free energy in the form of an equation for $\omega^{2}$ similar to the non-self-consistent equation (6.3):

$$
\omega^{2}=\left(\text { as in equation (6.3) with }\left\{\begin{array}{l}
v^{\mathrm{ps}} \rightarrow \tilde{v}^{\mathrm{ps}} \\
w \rightarrow \bar{w} \\
\varphi^{\mathrm{scr}} \rightarrow \tilde{\varphi}^{\mathrm{scr}}
\end{array}\right\}\right) .
$$

That is, $\omega^{2}$ has been expressed as a second derivative of a self-consistent potential (which involves $\omega^{2}$ itself via the smearing implicit in the potentials $\bar{w}$ and $\bar{\varphi}$ ).

Varying the free energy with respect to the $\{\boldsymbol{R}(l)\}$ one also obtains a condition that the self-consistent 'Kanzaki forces' are zero (see for example Matsubara and Kamiya 1977):

$$
0=F_{\mu}(l)=-\partial F^{\text {trial }} / \partial R_{\mu}(l)
$$

This equation will govern thermal expansion of the lattice. 
Putting the self-consistent solutions $\{\omega(l)\}$ and $\{\boldsymbol{R}(l)\}$ into (6.7) using (6.10)-(6.17) we obtain the optimal Einstein bound $F^{\text {trial,opt }}$ on the free energy of a simple metal system, be it a bulk or a surface configuration. This bound is rigorous to the extent that the effective potentials $w$ and $\varphi^{\text {scr }}$ of equations (2.8)-(2.10) are an adequate description of the lattice dynamical potential. The vibrational part of the free energy is given by subtracting the static energy (2.8) from the sum of (6.12), (6.11) and (6.10):

$$
\begin{aligned}
F^{\mathrm{vib}}(\text { self-consistent }) & =\operatorname{Tr} \sum_{l}\left\{\ln \left[\sinh \left(\frac{1}{2} \hbar \beta \omega(l)\right)\right]-\frac{1}{4} \hbar \omega(l) \operatorname{coth}\left(\frac{1}{2} \hbar \beta \omega(l)\right)\right\} \\
& +\sum_{l}[\tilde{w}(R(l))-w(R(l))] \\
& +\frac{1}{2} \sum_{l . l^{\prime}}\left[\hat{\varphi}^{\operatorname{scr}}\left(R(l), R\left(l^{\prime}\right)\right)-\varphi^{\operatorname{scr}}\left(R(l), R\left(l^{\prime}\right)\right)\right] .
\end{aligned}
$$

To obtain the vibrational contribution to the surface free energy, we subtract the bulk energy from that of two surface systems. This unfortunately means we have lost the rigorous variational character of the solution, if we treat both bulk and surface systems via (6.20). Nevertheless, such a model is sensible for two reasons:

(i) Because the trial free energy $F^{\text {trial }}$ is an extremum in the true thermal ensemble, it is relatively insensitive to departures from the correct ensemble. Thus our Einstein model gives not only a bound on the free energy, but also a reasonable approximation. (The approximation is worse for, say, the mean square excursion, which is not the extremised quantity.)

(ii) At very low temperatures where low-lying long-wavelength modes presumably invalidate the Einstein model, the vibrational free energy is only a small part of the total surface energy.

Thus finally we arrive at our self-consistent Einstein result for the total temperature-dependent surface free energy including some anharmonic effects:

$$
\sigma(T)=\sigma^{\text {static }}+(1 / 2 A)\left[2 F^{\mathrm{vib}}(\text { surf })-F^{\mathrm{vib}}(\text { bulk })\right]
$$

where $\sigma^{\text {static }}$ is given by $(3.14)$, and $F^{\mathrm{vib}}$ (surf) and $F^{\mathrm{vib}}$ (bulk) are the self-consistent solutions $(6.20)$.

\section{Summary and discussion}

We have obtained explicit $a b$ initio expressions for a number of quantities relating to simple metal surfaces, within electron density functional theory:

(i) We give the unscreened inhomogeneous surface electronic susceptibility $\chi^{0}\left(q, z, z^{\prime}\right)$ as an integral over real solutions $\psi_{p}^{+}(z), \psi_{p}^{c}(z)$ of the one-dimensional Lang-Kohn Schrödinger equation for a jellium surface. ( $\chi^{0}$ is defined by equations (A7) and (A19) of the Appendix, and is evaluated in equations (A31).)

(ii) The self-consistently screened surface electronic susceptibility $\chi\left(q_{\|}, z, z^{\prime}\right)$ is defined by equations (A32) and (A33), and can be obtained numerically by solving the integral equation (A38).

(iii) The $q \rightarrow \infty$ limiting behaviour of both $\chi^{0}\left(q, z, z^{\prime}\right)$ (equation A50) and $\chi\left(q !, z, z^{\prime}\right)$ (equations (A46) and (A48)) has been found.

(iv) We have proposed a simple ansatz

$$
\chi\left(q_{\|}, z, z^{\prime}\right) \sim \chi^{\text {bulk }}\left[q_{\mid}, z-z^{\prime}: \bar{n}=\frac{1}{2}\left(n(z)+n\left(z^{\prime}\right)\right)\right]
$$


for the self-consistently screened surface response in terms of the equivalent bulk quantity at an average electron density $\bar{n}$ determined from the zeroth-order densities at $z$ and $z^{\prime}$. We do not propose such an ansatz for the unscreened susceptibility, however. We have found that such an ansatz seems to work quite well, as tested so far on calculations of the total energy of systems containing discrete ions (see Chakravarty et al 1981, Rose and Dobson 1981). We do not, however, know at present how well the ansatz works when compared point-by-point with exact numerical values referred to in item (ii) above.

(v) We have expressed the total energy of an inhomogeneous metal (up to second order in the ion pseudopotentials) in terms of $\chi\left(r, r^{\prime}\right)$ and the ionic positions $\{X(l)\}$ (equation (2.8)).

(iv) We have used (v) to obtain an expression for the static $(T=0 \mathrm{~K})$ surface energy of any perfect face of a simple metal (equations (3.14)-(3.18)). We have evaluated this expression elsewhere (Rose and Dobson 1981), and find agreement with available experimental data across a range of simple metals and faces (see also paper II of this series).

(vii) We have obtained an expression for the linear static relaxation of ionic layer spacings near a simple metal surface (equations (4.8), (4.9) with (4.5) and (4.7)).

(viii) The surface dynamical matrix $D_{\alpha \beta}\left(\boldsymbol{q}, l, l^{\prime}\right)$ is given by equations (5.6)-(5.12). These results are similar to those of Inglesfield (1979), but refer to simple metals so that a much simpler susceptibility $\chi$ can be used.

(ix) The temperature-dependent harmonic vibrational contribution to the surface free energy is given approximately by equation (6.5) with the definitions (6.3). An anharmonic (self-consistent Einstein) result, which should yield improved results near melting, is given by (6.21) together with (6.20) and (6.12)-(6.15).

We defer discussion of our results (i)-(viii) to later papers in this series. We would, however, like to comment at this stage on a possible further use of the self-consistent Einstein lattice vibration calculation developed in $\S 6$ of the present paper.

In a previous publication (Dobson 1977) one of us noted that the smeared $t$ pair potential from the self-consistent Einstein approach can be substituted into the usual expression for the harmonic dynamical matrix, giving a modified matrix $D^{\text {sc }}$. The resultant phonon frequencies were shown, for the case of the soft modes of the quantum crystal metallic hydrogen, to agree quite well with the results of a full self-consistent phonon calculation incorporating anharmonic zero-point motion.

The relevance of this to the present work is that some metal surfaces may have temperature-dependent structural instabilities which will presumably be heralded by a softening of the surface phonon frequencies obtained from the self-consistent surface dynamical matrix $D_{\alpha \beta}^{\text {sc }}\left(q_{1}, l, l^{\prime}\right)$. See Trullinger and Cunningham (1973a, b) and Trullinger et al (1973). A good approximation to $D^{\text {sc }}$ in the case of large surface thermal motion may well be obtained by using our thermally smeared surface potentials (6.13) and (6.14) in our expressions (5.6) and (5.8) for the harmonic surface dynamical matrix. We believe this would be the first truly $a b$ initio theory of temperature-dependent surface reconstruction.

\section{Appendix. Formulae for self-consistently screened surface response function}

We consider electrons moving in an external potential $V_{+}^{\text {jell }}(z)$ due to a half-space of † 'Smearing' due to lattice motion should not be confused with 'screening' due to electron polarisation. All our ion-ion potentials are 'screened' via the screened electronic susceptibility $\chi$. 
uniform jellium background, plus an external three-dimensional perturbation $\Delta V_{\mathrm{ps}}(r)$ due to replacement of the jellium background by discrete pseudopotential ions. The self-consistent Kohn-Sham equation (Kohn and Sham 1965) is

$$
\left(-\frac{\hbar^{2}}{2 m} \nabla^{2}+V_{0}^{\mathrm{LK}}(z)+\Delta V^{\mathrm{sc}}(\boldsymbol{r})\right) \Psi_{k}(\boldsymbol{r})=\varepsilon_{k} \Psi_{k}(\boldsymbol{r}) .
$$

Here the total self-consistent potential energy is composed of external, exchangecorrelation and self-consistent Coulomb terms;

$$
\begin{gathered}
V_{0}^{\mathrm{LK}}(z)+\Delta V^{\mathrm{sc}}(r)=V_{-}^{\text {jell }}(z)+\Delta V_{\mathrm{ps}}(\boldsymbol{r})+\mu_{\mathrm{xc}}\left(n_{\mathrm{LK}}^{-}(z)+\Delta n(\boldsymbol{r})\right) \\
+e^{2} \int \mathrm{d}^{3} \boldsymbol{r}^{\prime} \frac{n_{\mathrm{LK}}^{-}\left(z^{\prime}\right)+\Delta n\left(\boldsymbol{r}^{\prime}\right)}{\left|r-\boldsymbol{r}^{\prime}\right|}
\end{gathered}
$$

where $\mu_{\mathrm{xc}}(n)$ is the exchange-correlation contribution to the chemical potential of a uniform gas of density $n$.

The total self-consistent electron density is

$$
n_{\mathrm{LK}}^{-}(z)+\Delta n(r)=2 \sum_{\varepsilon_{k}<\varepsilon_{\mp}} \Psi_{k}^{*}(r) \Psi_{k}(r)
$$

where $\varepsilon_{F}$ is the Fermi energy and the factor 2 accounts for spin orientations.

The zeroth-order problem is the one-dimensional jellium problem solved by Lang and Kohn (1970). It is characterised by the conditions $\Delta V_{\mathrm{ps}}=0=\Delta V^{\mathrm{sc}}, \Delta n=0$, with normalised wavefunctions

$$
\Psi_{k}^{0}(r)=(2 / \Omega)^{1 / 2} \exp \left[\mathrm{i}\left(k_{x} x+k_{y} y\right)\right] \psi_{k_{z}}(z)
$$

and eigenvalues

$$
\varepsilon_{\boldsymbol{k}}^{0}=\hbar^{2}|\boldsymbol{k}|^{2} / 2 m+\mu_{\mathrm{xc}}(\bar{n})+\varphi(-\infty)=\hbar^{2}|\boldsymbol{k}|^{2} / 2 m+V_{0}^{\mathrm{LK}}(-\infty) .
$$

The total zeroth-order self-consistent potential is $V_{0}^{\mathrm{LK}}(z)$. (Lang and Kohn (1970) designate it by $V_{\text {eff }}(n, z)$.) The wavefunction $\Psi_{k}^{0}$ in (A4) has been box-normalised in a large volume $\Omega=A \times L$, and the real one-dimensional Lang-Kohn wavefunctions have the following asymptotic behaviour deep inside the metal:

$$
\psi_{k}(z) \underset{z \rightarrow-x}{\sim} \sin [k z-\gamma(k)] .
$$

$\gamma(k)$ is a phaseshift introduced by Lang and Kohn $(1970), \mu_{x c}(\tilde{n})$ is the exchangecorrelation potential at $z \rightarrow-\infty$ where the density has the bulk value $\bar{n}$, and $\varphi(-\infty)$ is the electrostatic potential there.

\section{A.1. The unscreened response function}

We temporarily assume the self-consistent potential perturbation to be known, and to have a sinusoidal variation parallel to the metal surface:

$$
\Delta V^{\mathrm{sc}}(\boldsymbol{r})=\Delta V^{\mathrm{sc}}(z) \cos (\boldsymbol{q} \cdot \boldsymbol{r}), \quad \boldsymbol{q} \equiv q_{x} \boldsymbol{x}+q_{y} \boldsymbol{y} .
$$

The Schrödinger equation for the perturbed Kohn-Sham eigenfunctions is

$$
\left[-\left(\hbar^{2} / 2 m\right) \nabla^{2}+V_{0}^{\mathrm{LK}}(z)+\Delta V^{\mathrm{sc}}(z) \cos (\boldsymbol{q} \cdot r)\right] \Psi=\varepsilon \Psi .
$$

The first-order correction, $\delta \Psi$, to the zeroth-order Lang-Kohn solution $\Psi_{k}^{0}(r)$ satisfies

$$
\left[-\left(h^{2} / 2 m\right) \nabla^{2}+V_{0}^{\mathrm{LK}}(z)-\varepsilon_{k}^{0}\right] \delta \Psi_{k}(r)=-\Delta V^{\mathrm{sc}}(z) \cos (\boldsymbol{q} \cdot r) \Psi_{k}^{0}(\boldsymbol{r}) \text {. }
$$


The right-hand side of (A9) can be written

$$
\left.-(2 / \Omega)^{1 / 2} \frac{1}{2} \Delta V^{\mathrm{sc}}(z) \psi_{k_{z}}(z)\left\{\exp [\mathrm{i}(\boldsymbol{k}+\boldsymbol{q}) \cdot \boldsymbol{r}]+\exp \left[\mathrm{i}\left(\boldsymbol{k}_{\mid}-\boldsymbol{q}_{\|}\right) \cdot \boldsymbol{r}\right)\right]\right\} .
$$

Thus the wavefunction correction is

$$
\left.\delta \Psi_{k}(\boldsymbol{r})=(2 / \Omega)^{1 / 2} \frac{1}{2} \llbracket\left\{f_{k q}(z) \exp \left[\mathrm{i}\left(\boldsymbol{k}_{\mid}+\boldsymbol{q}_{\|}\right) \cdot \boldsymbol{r}\right]\right\}+\left\{\boldsymbol{q}_{\|} \rightarrow-\boldsymbol{q}_{\mid}\right\}\right]
$$

where $f_{k q}$ satisfies a one-dimensional Schrödinger equation:

$$
\left[\frac{\hbar^{2}}{2 m}\left(-\frac{\mathrm{d}^{2}}{\mathrm{~d} z^{2}}+\left(\boldsymbol{k}_{\|}+\boldsymbol{q}_{\|}\right)^{2}\right)+V_{0}^{\mathrm{LK}}(z)-\varepsilon_{k}^{0}\right] f_{k q}(z)=-\Delta V^{\mathrm{sc}}(z) \psi_{k_{z}}(z) .
$$

The solution of $(\mathrm{A} 11)$ is

$$
f_{k q \mid}(z)=\int_{-\infty}^{\infty} \mathrm{d} z^{\prime} G\left(z z^{\prime}, E_{k q \mid}\right) \Delta V^{\mathrm{sc}}\left(z^{\prime}\right) \psi_{k_{z}}\left(z^{\prime}\right)
$$

where

$$
E_{k q:}=\varepsilon_{k}^{0}-\left(\hbar^{2} / 2 m\right)\left(k_{\|}+q\right)^{2}
$$

and $G$ is the one-dimensional Green function satisfying

$$
\left(-\frac{\hbar^{2}}{2 m} \frac{\mathrm{d}^{2}}{\mathrm{~d} z^{2}}+V_{0}^{\mathrm{LK}}(z)-E\right) G\left(z, z^{\prime}, E\right)=-\delta\left(z-z^{\prime}\right) .
$$

The boundary conditions for (A14) must be specified. If we assume the perturbation to be switched on adiabatically from time $t=-\infty$, then causality demands that (A10) must represent waves travelling outward along the $z$ axis as $|z| \rightarrow \infty$, wherever the kinetic energy

$$
\left(\hbar^{2} / 2 m\right) p^{2}(z)=E-V_{0}^{\mathrm{LK}}(z)
$$

is positive. Where $p^{2}(z)$ is negative, the solution must decay as $|z| \rightarrow \infty$. The boundary conditions for (A14) are therefore

$$
\begin{aligned}
& G\left(z, z^{\prime}: E\right) \underset{z \rightarrow-\infty}{\sim} \begin{cases}\text { constant } \times \exp (-\mathrm{i}|p(-\infty)| z) & p^{2}(-\infty)>0 \\
\text { constant } \times \exp (+|p(-\infty)| z) & p^{2}(-\infty)<0,\end{cases} \\
& G\left(z, z^{\prime}, E\right) \underset{z \rightarrow+\infty}{\sim} \text { constant } \times \exp (-|p(+\infty)| z) .
\end{aligned}
$$

It should be noted that the energy (A13) can take on 'unphysical' values below the minimum value of the potential $V_{0}^{\mathrm{LK}}(z)$. In such cases the Green function is damped as $z \rightarrow-\infty$, though it is oscillatory there for the 'physical' range of energies. On the other hand, (A13) is always less than $\varepsilon_{\mathrm{F}}$ which ensures that the Green function is always damped as $z \rightarrow+\infty$, corresponding to the classically forbidden region outside the metal.

A linear expansion of (A3) gives the perturbation to the electronic number density as

$$
\Delta n(\boldsymbol{r})=4 \operatorname{Re}\left(\sum_{|k|<k_{\mathrm{F}}} \Psi_{k}^{0 *}(\boldsymbol{r}) \delta \Psi_{k}(\boldsymbol{r})\right)
$$

Using (A10) and (A12) we then obtain

$$
\Delta n(\boldsymbol{r})=\cos \left(\boldsymbol{q}_{\|} \cdot \boldsymbol{r}\right) \int \mathrm{d} z^{\prime} \chi^{0}\left(\boldsymbol{q}_{\|}, z, z^{\prime}\right) \Delta V^{\mathrm{SC}}\left(z^{\prime}\right)
$$


where the unscreened surface susceptibility is

$\chi^{0}\left(\boldsymbol{q}_{\mid}, z, z^{\prime}\right)=\frac{8}{(2 \pi)^{3}} \int_{\mid \boldsymbol{k}_{i}<k_{\mathrm{F}}} \mathrm{d}^{3} k \psi_{k_{z}}(z) \psi_{k_{z}}\left(z^{\prime}\right) \operatorname{Re} G\left(z, z^{\prime}, E=\varepsilon_{k}^{0}-\frac{\hbar^{2}}{2 m}(\boldsymbol{k} \mid \boldsymbol{q})^{2}\right)$.

There are two possible approaches when evaluating the one-dimensional Green function $G$. The first approach employs the eigenfunction expansion, with causality built in:

$$
G\left(z, z^{\prime}, E\right)=\lim _{\eta \rightarrow 0^{+}} \frac{2}{L} \sum_{k_{z}^{\prime}} \psi_{k_{z}^{\prime}}(z) \psi_{k_{z}^{\prime}}\left(z^{\prime}\right)\left(E-\varepsilon_{k_{z}}^{0}+\mathrm{i} \eta\right)^{-1} .
$$

Here $L$ is the length of the normalisation 'box', and the prefactor $(2 / L)$ arises because of the normalisation condition (A4). Using (A21) in (A20) we have

$\chi^{0}\left(\boldsymbol{q}, z, z^{\prime}\right)=\frac{16}{(2 \pi)^{3}} \lim _{\eta \rightarrow 0^{+}} \operatorname{Re} \frac{1}{L} \sum_{k_{z}^{\prime}} \int_{\mid k_{<}<k_{\mathrm{F}}} \mathrm{d}^{3} k \frac{\psi_{k_{z}}(z) \psi_{k_{z}}\left(z^{\prime}\right) \psi_{k_{z}}(z) \psi_{k_{z}^{\prime}}\left(z^{\prime}\right)}{\varepsilon_{k}^{0}-\varepsilon_{k_{1}+q_{1}, k_{z}}^{0}-\mathrm{i} \eta}$.

Here

$$
\varepsilon_{\boldsymbol{k}_{1}+\boldsymbol{q} \mid k_{z}}^{0}=\left(\hbar^{2} / 2 m\right)\left[\left(\boldsymbol{k}_{\|}+\boldsymbol{q}_{\|}\right)^{2}+k_{z}^{\prime 2}\right]
$$

The expression (A22) can also be derived directly from (A8) with the aid of timedependent first-order perturbation theory which naturally gives the imaginary term, i $\eta$, as a result of switching on the perturbation adiabatically. The $k$ integration in (A22) can be done analytically, yielding

$\chi^{0}\left(\boldsymbol{q} \mid, \boldsymbol{z}, \boldsymbol{z}^{\prime}\right)=\frac{8 m}{h^{2} q_{\mid}^{2} L} \sum_{k_{z}^{\prime}} \int_{-\infty}^{\infty} \mathrm{d} k_{z} \psi_{k_{z}}(z) \psi_{k_{z}}\left(z^{\prime}\right) \psi_{k_{z}}(z) \psi_{k_{z}^{\prime}}\left(z^{\prime}\right)(|\beta|-|\alpha|) S$

where

$$
\alpha=q^{2}+k_{z}^{\prime 2}-k_{z}^{2}, \quad S=\operatorname{sgn}(\alpha)
$$

and

$$
\beta^{2}=\max \left[0, \alpha^{2}-4 q^{2}\left(k_{\mathrm{F}}^{2}-k_{z}^{2}\right)\right]
$$

There are two difficulties with this approach. Firstly, the zeroth-order eigenfunctions $\psi_{k_{z}^{\prime}}$ are required for arbitrarily large energies. One can presumably avoid solving the Schrödinger equation numerically for very large energies, however, since the WKB approximation will be adequate when the kinetic energy is sufficiently dominant over the potential energy, so that the wavelength is very short. Secondly, the convergence of (A23a) does not appear to be very rapid.

The second approach to numerical evaluation of (A20), which was suggested to us by $\mathrm{E}$ Zaremba, proceeds by construction of the one-dimensional Green function $G\left(z, z^{\prime} ; E\right)$ out of 'left' and 'right' solutions $\psi_{p}^{+}(z), \psi_{p}^{-}(z)$ satisfying

$$
\left(-\frac{\hbar^{2}}{2 m} \frac{\mathrm{d}^{2}}{\mathrm{~d} z^{2}}+V_{0}^{\mathrm{i} \mathrm{K}}(z)-E\right) \psi_{p}^{z}(z)=0 \text {. }
$$

Like the Lang-Kohn eigenfunctions (A6), these solutions are labelled by their wavenumber deep inside the metal:

$$
p=p(-\infty)=(2 m)^{1 / 2} \hbar^{-1}\left(E-V_{0}^{\mathrm{LK}}(-\infty)\right)^{1 / 2} .
$$


Here, however, $p$ may be imaginary since (A20) calls for the Green function at a range of energies $E$ including 'unphysical' values below the minimum value of the potential $V_{0}^{\mathrm{LK}}(z)$.

The 'right' solution has the same boundary condition (A17) as the Green function, for $z \rightarrow \infty$ :

$$
\psi_{p}^{+}(z) \underset{z \rightarrow \infty}{\sim} \operatorname{constant} \times \mathrm{e}^{-\bar{X} z}
$$

where

$$
\bar{K}=(2 m)^{1 / 2} \hbar^{-1}\left(V_{0}^{\mathrm{LK}}(+\infty)-E\right)^{1 / 2}>0
$$

is the decay constant outside the metal. The 'constant' in (A25a) is chosen to make $\psi^{\top}$ agree with the Lang-Kohn eigenfunction, for real $p$ and $z \rightarrow-\infty$, while the choice is made for later convenience in the case $p^{2}<0$ :

$$
\psi_{p}^{+}(z) \underset{z \rightarrow-\infty}{\sim}\left\{\begin{array}{cl}
\sin \left(p z-\gamma_{p}\right) & p>0 \\
-\frac{1}{2} \exp (-|p| z) & p \text { imaginary. }
\end{array}\right.
$$

The 'left' solution $\psi_{p}^{-}$has the same boundary condition (A16) as the Green function, for $z \rightarrow-\infty$; we choose the following normalisation, involving the phaseshift $\gamma_{p}$ specified in (A6):

$$
\psi_{p}^{-}(z) \underset{z \rightarrow-\infty}{\sim} \begin{cases}\exp \left[-\mathrm{i}\left(p z-\gamma_{p}\right)\right] & p>0 \\ \exp (+|p| z) & p \text { imaginary. }\end{cases}
$$

The behaviour as $z \rightarrow+\infty$ is then a growing exponential:

$$
\psi_{p}^{-}(z) \underset{z \rightarrow+\infty}{\sim} \text { constant } \mathrm{e}^{+\bar{K} z}
$$

with $\bar{K}$ as in (A25b).

The Green function with the correct discontinuity in slope at $z=z^{\prime}$ can now be constructed in the usual fashion:

$$
G\left(z, z^{\prime}, E\right)=\frac{2 m}{\hbar^{2}} \frac{\psi_{p}^{+}\left(z_{>}\right) \psi_{p}^{-}\left(z_{<}\right)}{W\left(\psi_{p}^{+}, \psi_{p}^{-}\right)} .
$$

Here $z_{>}=\max \left(z, z^{\prime}\right), z_{<}=\min \left(z, z^{\prime}\right)$ and $W(f, g)=f \mathrm{~d} g / \mathrm{d} z-g \mathrm{~d} f / \mathrm{d} z$ is the Wronskian of two functions. From the Schrödinger equation (A24a) it is easily shown that $W\left(\psi^{+}, \psi^{-}\right)$is independent of $z$, for two solutions $\psi^{ \pm}$of the same energy. Thus we may evaluate $W$ from the $z \rightarrow-\infty$ forms of $\psi^{+}$and $\psi^{-}$:

$$
W\left(\psi_{p}^{+}, \psi_{p}^{-}\right)=-|p| .
$$

The fact that $W$ has the same form for real and imaginary $p$ is not automatic, but was ensured by careful choice of the asymptotic forms (A25c) and (A26a). In evaluating the response function (A20) we require the real part of $G$ which, from (A27) and (A28) is

$$
\operatorname{Re} G\left(z, z^{\prime}, E\right)=\left(2 m / \hbar^{2}|p|\right) \psi_{p}^{+}\left(z_{>}\right) \psi_{p}^{c}\left(z_{<}\right) .
$$

Here $\psi_{p}^{f}(z)$ is the real part of $\psi_{p}^{-}(z)$. It satisfies (A24a) with the boundary condition

$$
\psi_{p}^{c}(z) \underset{z \rightarrow-\infty}{\sim} \begin{cases}\cos \left(p z-\gamma_{p}\right) & p>0 \\ \exp (|p| z) & p \text { imaginary. }\end{cases}
$$

Note that, for 'physical' energies $\varepsilon_{\mathrm{F}}>E>V_{0}^{\mathrm{L} K}(-\infty)$ corresponding to real values of $p$, 
$\psi_{p}^{c}$ is the 'cosine' solution complementary to the Lang-Kohn eigenfunction $\psi_{p}^{+}$(the 'sine' solution).

The unscreened surface response function is now found from (A29), (A24b) and (A20):

$$
\begin{aligned}
\chi^{0}\left(\boldsymbol{q}_{i}, z, z^{\prime}\right)=\frac{16 m}{(2 \pi)^{3} \hbar^{2}} \int_{|k|<k_{F}} \mathrm{~d}^{3} k \psi_{k_{2}}^{+}(z) \psi_{k_{z}}^{+}\left(z^{\prime}\right) \frac{\psi_{p}^{+}\left(z_{>}\right) \psi_{p}^{c}\left(z_{<}\right)}{|p|} \\
=\frac{32 m}{(2 \pi)^{3} \hbar^{2}} \int_{k_{\bar{z}}+k_{\mathbf{z}}<k^{2}} \mathrm{~d} k_{x} \mathrm{~d} k_{z}\left[k_{\mathrm{F}}^{2}-k_{x}^{2}-k_{z}^{2}\right]^{1 / 2} \frac{\psi_{k_{z}}^{+}(z) \psi_{k_{z}}^{+}\left(z^{\prime}\right) \psi_{p}^{+}\left(z_{>}\right) \psi_{p}^{c}\left(z_{<}\right)}{|p|}
\end{aligned}
$$

where

$$
p^{2} \equiv p^{2}(-\infty)=k_{z}^{2}-2 k_{x} q_{\|}-q_{!}^{2}
$$

(see equation (A20)), and $\psi_{k}^{+}$and $\psi_{k}^{c}$ are solutions of the Schrödinger equation (A24) with energy

$$
E=V_{0}^{\mathrm{LK}}(-\infty)+\left(h^{2} / 2 m\right) k^{2}
$$

and boundary conditions (A25) and (A30); these solutions must, in general, be obtained numerically.

\section{A.2. The self-consistent screening equation}

The unscreened susceptibility $\chi^{0}$ was defined as the density response to the total (screened) potential $\Delta V^{\text {sc }}$. We now define the self-consistent (screened) susceptibility $\chi\left(q_{\|}, z, z^{\prime}\right)$ in terms of the linear electron density perturbation resulting from an external (unscreened) potential $\Delta V_{\mathrm{ps}}$ localised at $z^{\prime}$ on the $z$ axis and varying sinusoidally in the $x y$ plane.

Thus if

$$
\Delta V_{\mathrm{ps}}(\boldsymbol{r})=\alpha \delta\left(z-z^{\prime}\right) \cos \left(\boldsymbol{q}_{\mid} \cdot \boldsymbol{r}\right) \quad \alpha \rightarrow 0
$$

then

$$
\Delta n(\boldsymbol{r})=\alpha \chi\left(q_{\mid}, z, z^{\prime}\right) \cos \left(\boldsymbol{q}_{\mid} \cdot \boldsymbol{r}\right)+\mathrm{O}\left(\alpha^{2}\right) .
$$

To find an expression for $\chi\left(q \|, z, z^{\prime}\right)$ we linearise the self-consistency equation (A2), obtaining

$$
\Delta V^{\mathrm{xc}}\left(\boldsymbol{r}_{1}\right)=\Delta V_{\mathrm{ps}}\left(\boldsymbol{r}_{1}\right)+\mu_{\mathrm{xc}}^{\prime}\left(z_{1}\right) \Delta n\left(\boldsymbol{r}_{1}\right)+e^{2} \int \mathrm{d}^{3} r_{2} \frac{\Delta n\left(\boldsymbol{r}_{2}\right)}{\left|\boldsymbol{r}_{1}-\boldsymbol{r}_{2}\right|}
$$

where

$$
\mu_{\mathrm{xc}}^{\prime}(z)=\left.\frac{\mathrm{d} \mu_{\mathrm{xc}}(n)}{\mathrm{d} n}\right|_{n=n \overline{\mathrm{L}} \mathrm{K}(z)} .
$$

Substituting (A33) and (A32) into (A34) and performing the surface part, $\int \mathrm{d} x_{2} \mathrm{~d} y_{2}$, of the $r_{2}$ integral, we find

$$
\begin{aligned}
\Delta V^{\mathrm{sc}}\left(\boldsymbol{r}_{1}\right)=\alpha & \cos \left(\boldsymbol{q}_{\|} \cdot \boldsymbol{r}_{1}\right)\left(\delta\left(z_{1}-z^{\prime}\right)+\mu_{\mathrm{xc}}^{\prime}\left(z_{1}\right) \chi\left(q_{\|}, z_{1}, z^{\prime}\right)\right. \\
& \left.+2 \pi e^{2} \int \frac{\exp \left(-q_{\|}\left|z_{1}-z_{2}\right|\right)}{q_{\|}} \chi\left(q_{\|}, z_{2}, z^{\prime}\right) \mathrm{d} z_{2}\right) .
\end{aligned}
$$


We now multiply throughout by $\chi^{0}\left(q_{\|}, z, z_{1}\right)$ and integrate with respect to $z_{1}$. Then, after using (A19) and (A33), we can cancel $\alpha \cos (q \mid \cdot r)$ throughout to give the integral equation for the screened susceptibility:

$$
\chi\left(q_{\sharp}, z, z^{\prime}\right)=\chi^{0}\left(q_{\|}, z, z^{\prime}\right)+\int \mathrm{d} z_{1} Q\left(q_{\|}, z, z_{1}\right) \chi\left(q_{\|}, z_{1}, z^{\prime}\right)
$$

where

$Q\left(q_{\|}, z, z_{1}\right)=\mu_{\mathrm{xc}}^{\prime}\left(z_{1}\right) \chi^{0}\left(q_{\|}, z, z_{1}\right)+2 \pi e^{2} \int \mathrm{d} z_{2} \chi^{0}\left(q_{\|}, z, z_{2}\right) \frac{\exp \left(-q_{\|}\left|z_{1}-z_{2}\right|\right)}{q_{\|}}$.

Equation (A37) must be solved numerically (e.g. by discretising it and using matrix inversion). Analytic solution is not possible because $\chi^{0}$ lacks translational invariance. (Note that in (A36) and (A38) the quantity $\exp \left(-q \| z_{1}-z_{2} \mid\right) / q_{\|}$is to be replaced by $-\left|z_{1}-z_{2}\right|$ if $q_{\|}=0$.)

\section{A.3. Tests and limiting cases}

To see the kind of behaviour embodied in the unscreened response formula (A31) we consider the artificial case of non-interacting electrons confined to the half-space $z<0$. Thus we fix the zeroth-order potential as

$$
V_{0}^{\mathrm{LK}}(z)= \begin{cases}\infty & z>0 \\ 0 & z<0 .\end{cases}
$$

Then, for $z<0$,

$$
\begin{aligned}
& \psi_{k}^{+}(z)= \begin{cases}\sin (k z) & k>0 \\
\sinh (|k| z) & k \text { imaginary }\end{cases} \\
& \psi_{k}^{\leftarrow}(z)= \begin{cases}\cos (k z) & k>0 \\
\exp (|k| z) & k \text { imaginary } .\end{cases}
\end{aligned}
$$

Using product-to-sum trigonometric identities in (A31) we obtain a particularly simple result when $q_{\|}=0$ :

$$
\begin{aligned}
\chi\left(q_{\|}=0, z, z^{\prime}\right) & =\chi^{0(\mathrm{~L})}\left(\left|z-z^{\prime}\right|\right)+\chi^{0(\mathrm{~L})}\left(\left|z+z^{\prime}\right|\right) \\
& -2 \chi^{0(\mathrm{~L})}\left(\left(\left|z-z^{\prime}\right|+\left|z+z^{\prime}\right|\right) / 2\right) .
\end{aligned}
$$

Here

$$
\chi^{O(\mathrm{~L})}(z)=\frac{4 m}{(2 \pi)^{3} \hbar^{2}}\left(\int_{k<k_{\mathrm{K}}} \mathrm{d}^{3} k \frac{\cos \left(k_{z} z\right) \sin \left(k_{z}|z|\right)}{k_{z}}-\pi^{2} k_{\mathrm{F}}^{2}\right)
$$

is readily shown to be the one-dimensional Fourier transform of the Lindhard function $\chi^{(\mathrm{L})}(q)($ Lindhard 1954):

$$
\begin{aligned}
& \chi^{0(\mathrm{~L})}(z)=\frac{1}{2 \pi} \int_{-\infty}^{\infty} \mathrm{d} q \mathrm{e}^{\mathrm{i} q z} \chi^{0(\mathrm{~L})}(q) \\
& \chi^{0(\mathrm{~L})}(q)=\frac{8 m}{(2 \pi)^{3} \hbar^{2}} \int_{k<k_{\mathrm{F}}} \mathrm{d}^{3} k \mathrm{P}\left(\frac{1}{k_{z}^{2}-\left(k_{z}+q\right)^{2}}\right) .
\end{aligned}
$$

The first term in (A40) represents bulk-style response due to electrons moving directly from disturbance $z^{\prime}$ to reception at $z$; the second term represents electrons reflected off 
the infinite potential barrier before reception. The third term ensures that $\chi=0$ if $z$ or $z^{\prime}=0$, a necessary property since the unperturbed eigenfunctions vanish there. This third term is the 'interference' term of Garrido et al (1979). After some algebra it can also be related to the 'non-diagonal response' $A_{Q, q, q^{\prime}}(\omega=0)$ of Newns (1970). For $q \mid \neq 0$ the result is not so simple, but the same basic physics emerges.

With the more realistic smooth self-consistent jellium potential $V_{0}^{\mathrm{LK}}(z)$, there is no one place (such as $z=0$, as above) where electrons all suffer reflection, so the type of behaviour described above for the infinite-barrier problem could be smeared. By performing an asymptotic expansion of (A31) using (A25c) and (A30) we have shown, however, that 'reflected Friedel oscillations' persist in $\chi^{0}\left(q_{i}, z, z^{\prime}\right)$ for values of $z$ well inside the bulk, even though $V_{\mathrm{LK}}^{0}(z)$ is smooth. This is due to reflection of Fermi surface electrons at a well defined location.

Although the response formula (A31) and the screening equation (A37)-(A38) must be treated numerically in general, an approximate analytic solution is available when $q \gg 2 k_{\mathrm{F}}$. In this case the 'right' and 'left' solutions $\psi_{p}^{+}, \psi_{p}^{f}$ appearing in (A31) may be evaluated in the WKB approximation, because the 'wavelength' $|p(z)|^{-1}$ (equation (A15)) is always much shorter than the scale length $k_{\mathrm{F}}^{-1}$ of the potential $V_{0}^{\mathrm{t} K}$ (see also equation (A31c)). The WKB wavefunctions satisfying (A25c) and (A30), for the imaginary values of $p$ which are relevant here, are

$$
\begin{aligned}
& \psi_{p}^{+}(z)=-\frac{1}{2} \exp \left(-|p| z_{0}\right)\left|\frac{p}{p(z)}\right|^{1 / 2} \exp \left(-\int_{z_{0}}^{z}\left|p\left(z^{\prime}\right)\right| \mathrm{d} z^{\prime}\right) \\
& \psi_{p}^{\mathcal{f}}(z)=\exp \left(|p| z_{0}\right)\left|\frac{p}{p(z)}\right|^{1 / 2} \exp \left(+\int_{z_{0}}^{z}\left|p\left(z^{\prime}\right)\right| \mathrm{d} z^{\prime}\right)
\end{aligned}
$$

where $z_{0}$ is a point well inside the metal so that $V_{0}^{\mathrm{L}}\left(z_{0}\right) \sim V_{0}^{\mathrm{L} K}(-\infty)$. The quantity $p(z)$ is defined in equation (A15). Thus

$$
\frac{\psi_{p}^{+}\left(z_{>}\right) \psi_{p}^{\hat{c}}\left(z_{<}\right)}{|p|}=-\frac{\exp \left(-\int_{z_{<}}^{z_{>}}\left|p\left(z^{\prime}\right)\right| \mathrm{d} z^{\prime}\right)}{2\left|p\left(z_{>}\right) p\left(z_{<}\right)\right|^{1 / 2}}
$$

Since $\psi_{k_{z}}(z)$ in (A31) is an occupied physical orbital, it cannot vary on a spatial scale smaller than about $k_{\mathrm{F}}^{-1}$. (The decay constant outside the metal does not greatly exceed $k_{\mathrm{F}}$ so the above statement holds for all $z$.) For $q \gg 2 k_{\mathrm{F}}$, the exponential in (A44) cuts off on a much smaller scale and accordingly, for purposes of evaluating (A31), we approximate it by a delta-function:

$$
\begin{gathered}
\psi_{p}^{+}\left(z_{>}\right) \psi_{p}^{c}\left(z_{<}\right) /|p| \underset{q \gg 2 k_{F}}{\sim}-\frac{1}{2}\left|p\left(z_{>}\right)\right|^{-1} \exp \left[-\left|p\left(z_{>}\right)\right|\left(z_{>}-z_{<}\right)\right] \\
\sim-\delta\left(z_{>}-z_{<}\right) /\left|p^{2}\left(z_{>}\right)\right| \sim-\delta\left(z_{>}-z_{<}\right) / q^{2} .
\end{gathered}
$$

Thus the response function (A31a) becomes local and depends on the wavefunctions only through the density:

$$
\begin{aligned}
\chi^{0}\left(\boldsymbol{q} \|, z, z^{\prime}\right) \underset{q: \gg 2 k_{\mathrm{F}}}{\sim}-\frac{16 m \delta\left(z-z^{\prime}\right)}{(2 \pi)^{3} \hbar^{2} q_{\|}^{2}} \int_{k<k_{\mathrm{F}}} \mathrm{d}^{3} k \psi_{k_{z}}^{+2}(z) \\
=-\frac{4 m}{\hbar^{2} q_{\|}^{2}} n_{\mathrm{LK}}^{-}(z) \delta\left(z-z^{\prime}\right) .
\end{aligned}
$$


This will be an adequate approximation when one integrates $\chi^{0}$ with a slowly varying $\left(\lambda \geq k_{\mathrm{F}}^{-1}\right)$ function of $z$.

In the same limit $q_{\|} \gg 2 k_{\mathrm{F}}$, the screening kernel $Q$ of equation (A38) becomes

$Q\left(q \|, z, z_{1}\right) \underset{q \gg 2 k_{\mathrm{F}}}{\sim}-\frac{4 m n_{\mathrm{LK}}^{-}(z)}{\hbar^{2} q_{\|}^{2}}\left(\mu_{\mathrm{xc}}^{\prime}(z) \delta\left(z-z_{1}\right)+\frac{2 \pi e^{2}}{q_{\mid}} \exp \left(-q_{\|}\left|z-z_{1}\right|\right)\right)$.

Replacing the exponential in (A47) by a delta-function as before, we have a local kernel and so (A37) can be solved giving

$$
\chi\left(q_{\|}, z, z^{\prime}\right) \underset{q: 2 k_{\mathrm{F}}}{\sim}-\frac{A\left(q_{\|}, z\right)}{1+\left(\mu_{\mathrm{xc}}^{\prime}(z)+4 \pi e^{2} / q_{\|}^{2}\right) A\left(q_{\|}, z\right)} \delta\left(z-z^{\prime}\right)
$$

where

$$
A(q \mid, z)=4 m n_{\mathrm{LK}}^{-}(z) / \hbar^{2} q_{\|}^{2}
$$

We stress that (A48) is valid in the context of integration with functions of $z$ whose scale length is $k_{\mathrm{F}}^{-1}$ or longer. It is also apparent that the screening becomes ineffective at very large $q_{\|}$.

\section{Acknowledgments}

The authors would like to thank E Zaremba, L Sander and J Perdew for useful discussions. J Perdew, K P Bohnen, S C Ying and A Zunger also kindly provided preprints and/or data prior to publication.

JFD would like to thank the staff of the Physics Department, University of Michigan, for hospitality during a five-month visit during 1980 .

This work was supported in part at the University of Michigan by NSF grant DMR-78-25012. Ames Laboratory is operated for the US Department of Energy by Iowa State University under contract No W-7405-ENG-82. JHR's work there was supported by the Director of Energy Research, Office of Basic Sciences.

\section{References}

Allen R E and De Wette F W 1969 J. Chem. Phys. $514820-9$ Ashcroft N W and Langreth D C 1967 Phys. Rev. 155 682-4 Bohnen K-P and Ying S C 1979 Solid State Commun. 30 301-4 — 1980 Phys. Rev. B 22 1806-17

Chakravarty S, Rose J H, Wood D M and Ashcroft N W 1981 Phys. Rev. B 24 1624-35

Dobson J F 1977 Phys. Lett. A 62 368-70

Dobson J F and Ashcroft N W 1977 Phys. Rev. B $165326-40$

Feynman R P 1972 Statistical Mechanics (Reading, MA: Benjamin)

Finnis N W 1974 J. Phys. F: Met. Phys. 4 1645-56 1975 J. Phys. F: Met. Phys. 5 2227-40

Garrido L M, Flores F and Garcia-Moliner F 1979 J. Phys. F: Met. Phys. 9 1097-110

Hammerberg J and Ashcroft N W 1974 Phys. Rev. B $9409-24$

Hohenburg P and Kohn W 1964 Phys. Rev. 136 B864-71

Inglesfield J E 1979 J. Phys. C: Solid State Phys. 12 149-65

Kittel C 1963 Quantum Theory of Solids (New York: Wiley) p 109

Kohn W and Sham L J 1965 Phys. Rev. 140 A1133-8

Landman U, Hill R N and Mostoller M 1980 Phys. Rev. B 21 448-57 
Lang N D and Kohn W 1970 Phys. Rev. B $14555-68$

Lindhard J $1954 \mathrm{Kgl}$. Danske Vidensk. Selsk. Mat.-Fys. Meddr, 28 number 8

Lert P W and Weare J H 1978 J. Chem. Phys. 68 5010-9

Maradudin A A, Montroll E W, Weiss G H and Ipatova I P 1971 Theory of Lattice Dynamics in the Harmonic Approximation 2nd edn (New York: Academic Press)

Matsubara T and Kamiya K 1977 Prog. Theor. Phys. 58 767-76

Messiah A 1965 Quantum Mechanics Part I (Amsterdam: North-Holland)

Monnier R and Perdew J P 1978 Phys. Rev. B 17 2595-611 (Erratum 198022 1124)

Newns D M 1970 Phys. Rev. B 13304-22

Paasch G and Hietschold M 1977 Phys. Status Solidi b 83 209-22

Perdew J P 1982 Phys. Rev. B $106291-9$

Perdew J P and Monnier R 1980 J. Phys. F: Met. Phys. 10 L287-91

Rose J H and Dobson J F 1981 Solid State Commun. 37 91-6

Stott M J and Zaremba E 1980 Phys. Rev. A 21 12-23

Trullinger S E and Cunningham S L 1973a Phys. Rev. B 8 2622-38

- 1973b Phys. Rev. Lett. 30 913-6

Trullinger S E, Cunningham S L and Mills D L 1973 Solid State Commun. 13 975-8

Tyson W R and Miller W A 1977 Surf. Sci. 62 267-76

Wawra H 1975a Z. Metallkunde 66 395-401

- 1975b Z. Metallkunde 66 492-8

Ying S C, Smith J R and Kohn W 1972 J. Vac. Sci. Technol. 9 575-8 